\title{
Neurotoxicity of zinc oxide nanoparticles and municipal effluents to Fathead minnows
}

\author{
Gagné F*, Auclair J, Pilote M, Turcotte P and Gagnon C \\ Aquatic Contaminants Research Division, Water Science and Technology, Environment Canada, 105 McGill, Montreal, Quebec, Canada
}

\begin{abstract}
Nanotechnology product development is an area of intense commercial activity and is raising concerns about the possible impacts on the environment. Zinc oxide nanoparticles (nano-ZnO), which are used as transparent sunscreens and cosmetics, find their way into the aquatic environment via surface water runoff near beaches and wastewater treatment plants. The purpose of this study was to examine the neurotoxicity of municipal effluent in fathead minnows exposed to either dietary nano$\mathrm{ZnO}$ or $\mathrm{ZnCl}_{2}$. Adult male and female fathead minnows were exposed to $100 \mathrm{ng} \mathrm{Zn} / \mathrm{g}$ of either nano- $\mathrm{ZnO}$ or $\mathrm{ZnCl} \mathrm{in}_{2}$ the diet and to dilutions of municipal effluent for 21 days at $25^{\circ} \mathrm{C}$. The following biomarkers were determined after the exposure period: tissue metal levels, brain DNA damage, lipid peroxidation, metallothioneins, dopamine, acetylcholinesterase, monoamine oxidase and mitochondrial electron transport activity (a marker of cellular energy expenditure). The data revealed that although fish did not significantly accumulate $\mathrm{Zn}$ from dietary nano- $\mathrm{ZnO}$ or $\mathrm{ZnCl}_{2}$, the difference in tissue levels of $\mathrm{Zn}$ was significantly higher in females than in males when nano- $\mathrm{ZnO}$ or $\mathrm{ZnCl} 2$ was added in the presence of municipal effluent. Monoamine oxidase and acetylcholinesterase activity and dopamine levels were induced by the municipal effluent, but was halted by the addition of both dietary $\mathrm{Zn}$ forms. Exposure of fish to dietary nano-ZnO led to more significant changes in mitochondrial electron transport activity, acetylcholinesterase activity, and dopamine and $\mathrm{MT}_{\text {levels, while exposure to dietary } \mathrm{ZnCl}}$ and municipal effluent led to additional changes in gonadosomatic index, dopamine, $\mathrm{Ag}$ and $\mathrm{Cu}$ levels. Increased brain LPO levels were reported in males exposed to the municipal effluent and dietary nano-ZnO. Decreased DNA strand breaks were observed in fathead minnows exposed to the municipal effluent, suggesting decreased DNA repair activity, but were not observed in the presence of dietary nano- $\mathrm{ZnO}$ and $\mathrm{ZnCl}_{2}$. In conclusion, the neurotoxicological properties of the municipal effluents could be modulated by dietary nano- $\mathrm{ZnO}$ or $\mathrm{ZnCl}_{2}$, and in the presence of municipal effluent, nano- $\mathrm{ZnO}$ effects were closely related to those in fish fed a $\mathrm{ZnCl}$-supplemented diet.
\end{abstract}

\section{Introduction}

Nanotechnology is the production of engineered materials in the size range of 1-100 $\mathrm{nm}$. The colloidal nature of these products brings about high specific surface areas with emerging physicochemical properties that are not observed from their constituents. The current development of the industry raises the issue of inadvertent releases of nanomaterials into the environment with unknown consequences on wildlife. Information on their persistence and stability in the environment is currently available [1]. Zinc oxide nanoparticles (nano$\mathrm{ZnO}$ ) are used in a wide variety of commercial applications, including personal care products, such as transparent sunscreens, as well as in cosmetics, ointments, pigments and lubricants because of their antibacterial properties [2]. The antibacterial activity of nano- $\mathrm{ZnO}$ is of added interest to consumers, who depend on the "controlled" release of $\mathrm{Zn}^{2+}$ ions in cream preparations [3, 4]. Uncoated nano- $\mathrm{ZnO}$ has low zeta potential, which makes them susceptible to aggregation and precipitation in solution [5]. The presence of dissolved organic material (DOM), which is found at relatively high concentrations in some types of surface water (brown water) and municipal effluents, could limit aggregation and increase the proportion of the monomeric form of nano- $\mathrm{ZnO}$ in solution [6]. The increasing use of nano-ZnO-based sunscreens and cosmetics has raised the issue of the contamination of surface waters in highly populated areas with recreational beaches and their release by municipal effluents. The interaction between nano- $\mathrm{ZnO}$ and the organic matrix of municipal wastewaters is little understood at this time.

The effects of the toxicity of municipal effluents, which are highly complex mixtures, are diverse and include endocrine disruption, genotoxicity, inflammation and oxidative stress [7-9]. Municipal effluents are known to act as endocrine disruptors, affecting reproduction by interfering with estrogen and serotonin receptor pathways $[10,11]$. They also contain hormones and pharmaceuticals (17a-ethinyl-estradiol), which can contribute to disruption of the sexual differentiation, gametogenesis and egg maturation. In a previous study, wild sexually immature spottail shiners collected downstream of a major municipal effluent outfall in the St. Lawrence River (QC, Canada) expressed vitellogenin mRNA, which codes for vitellogenin, the main egg yolk protein precursor normally expressed in females [12]. In addition, spermatogenesis was delayed in fish collected downstream where sperm counts and motility were significantly lower. The prevalence of intersex, a condition whereby males exhibit female oocytes, were above the background levels at $30 \%$ at the downstream sites. Serotonergic effects in trout exposed to municipal effluents were also observed, and serotonergic activity (serotonin-dependent ATPase activity in brain synaptosome) was correlated with brain tissue concentrations of some selective serotonin reuptake inhibitors [13].

These studies suggest that endocrine disruption that occurs in fish could resonate at the neurological level as well. The neurotoxicological

Correspondence to: François Gagne, Aquatic Contaminants Research Division, Water Science and Technology, Environment and Climate Change Canada, 105 McGill, Montreal, QC, Canada; E-mail: francois.gagne@canada.ca

Keywords: Fathead minnows, municipal effluents, zinc oxide nanoparticles, neurotoxicity, DNA damage, oxidative stress and energy expenditure

Received: December 10, 2015; Accepted: January 20, 2016; Published: Janaury 25,2016 
properties of municipal effluents, which are sources of neuroactive agents (pharmaceuticals), estrogens and nanoparticles in the aquatic environment, in fish are not well understood. Fathead minnows exposed to municipal effluents were found to have induced plasma vitellogenin and evidence of ovipositors in male fish [14]. The secondary sexual characteristics of male were less apparent as evidenced by discoloration of the banding patterns, little development of the dorsal pad and absence of a dorsal fin dot. Nanoparticles and municipal effluents could also lead to oxidative stress and genotoxicity [15-17]. Nerve tissues are rich in polyunsaturated lipids, which can undergo lipid peroxidation (LPO) in the presence of reactive oxygen species. Indeed, hepatic LPO was significantly elevated in organisms found in the vicinity of sewage pollution (Bianchi et al., 2014). Oxidative stress could also lead to DNA damage by the formation of oxygen adducts (8-oxo-guanosine). Evidence of DNA damage in fish exposed to municipal effluents and to nanoparticles with surface area reactivity has been reported $[16,17]$. Metallothioneins (MT) a small molecular weight cysteinerich proteins that bind essential divalent elements such $\mathrm{Zn}$ and copper $(\mathrm{Cu})$ and non-essential elements such as cadmium, mercury and silver [18]. The MT biomarker provides useful information on the the presence and mobility of metal ions in cells. Neural activity could be monitored by acetylcholinesterase (AchE) activity, which is involved in the inactivation of acetylcholine, an important neurotransmitter in the central nervous system that is involved in learning, physiological stimulation and plasticity $[19,20]$. Monoamine oxidases (MAO) are located in the mitochondria and involved in the oxidative deamination of indoleamines (serotonin) and catecholamines (dopamine and adrenaline), which are major neurotransmitters in the brain. Catecholamines are considered stimulatory neurotransmitters involved in the activation of $\beta$-adrenergic signaling (sympathetic state) and feeding reward [21]. It has been shown that in fish under $\beta$-adrenergic stimulation, high levels of dopamine were associated with conditioned place preference paradigm behaviour (the preference of fish to stay or visit a tile for egg laying for example) and this response could be modulated by central cholinergic stimulation [22]. Decreased acetylcholinesterase activity was shown to reduce conditioned place preference behaviour, in fish suggesting an interaction between monoamine stimulation and cholinergic signalization in the brain. Neural activity is also coupled with metabolic energy expenditure, which can be determined by mitochondrial electron transport activity, which is associated with $\mathrm{O}_{2}$ consumption and $\mathrm{CO}_{2}$ production in cells, which requires glucose for ATP production. In a previous study, elevated mitochondrial electron transport (MET) activity in visceral mass was observed in mussels exposed to municipal effluents, suggesting increased activity and energy expenditure. This suggests that aquatic organisms exposed to urban pollutants expend more energy than organisms from more pristine sites, which can compromise health status, growth and reproduction in the long term.

The purpose of this study was to examine the neurotoxicity of combined exposures to dietary nano- $\mathrm{ZnO}$ or $\mathrm{ZnCl}_{2}$ and municipal effluent in adult fathead minnows. Fathead minnows were exposed to nano- $\mathrm{ZnO}$ or $\mathrm{ZnCl}_{2}$ though their diets and to dilutions of a typical physicochemically treated effluent. The dietary route exposure for nano- $\mathrm{ZnO}$ was selected given the poor solubility of uncoated $\mathrm{ZnO}$ in water and to ensure that the fish were exposed to the nanoparticles present in the feed. For comparison purposes, fish were also exposed to dietary $\mathrm{ZnCl}_{2}$ to determine whether or not the same interaction with the municipal effluent and nano- $\mathrm{ZnO}$ diet occurred. The bioavailability of $\mathrm{Zn}$ was determined in fish carcasses, and biomarkers of stress were determined in brain tissue. The biomarkers were MT, LPO and DNA damage. Neurotoxicity was assessed by measuring changes in AchE, $\mathrm{MAO}$ and dopamine levels and mitochondrial electron transport activity. An attempt was made to highlight the cumulative effects of a physicochemically treated municipal effluent and dietary nano- $\mathrm{ZnO}$ or $\mathrm{ZnCl}_{2}$ for 21 days based on sex, municipal effluent concentrations and $\mathrm{Zn}$ forms in adult fathead minnows.

\section{Materials and methods}

\section{Fathead minnow reproduction assay and exposure to municipal effluent}

Fish were cultured and bred at a fathead minnow colony at the wet laboratory of the Aquatic toxicology laboratory at the Montreal Wastewater Treatment Plant (QC, Canada). The study employed a 21day exposure regime with a $1: 2$ male to female ratio in $12.5 \mathrm{~L}$ tanks into which diluted municipal effluents was continuously pumped (1 $\mathrm{L}$ per hour). Briefly, two males and four females were held in a 12-L aquarium for a period of 7 days prior to exposure to the effluent, dietary $\mathrm{ZnCl}_{2}$ and nano- $\mathrm{ZnO}$. The aquariums contained spawning tiles made from two $8-\mathrm{cm}$ lengths of polyvinyl pipes with a $10-\mathrm{cm}$ diameter, cut in half longitudinally, which were monitored in the mornings for egg production. Successful fertilization of spawned eggs under a microscope, and groups exhibiting the highest egg fertilization rate were selected for the experiment. In fully active fish, exposure to the effluent was initiated using concentrations of 5,10 and $20 \% \mathrm{v} / \mathrm{v}$ at $25^{\circ} \mathrm{C}$. The effluent was constantly renewed $(1 \mathrm{~L} / \mathrm{hr}$ flow rate) and was pre-heated to $25^{\circ} \mathrm{C}$ before being pumped into the fish aquariums. The exposure experiments were repeated with two replicate tanks for each treatment group. In addition to real-time exposure to the municipal effluents, one group of fish was exposed to either nano- $\mathrm{ZnO}$ - or $\mathrm{ZnCl}_{2}$-spiked feed at a nominal concentration of $100 \mu \mathrm{g}$ of total $\mathrm{Zn}$ per $\mathrm{kg}$ of feed. The fish were fed daily with commercial feed during the exposure experiments ( $10 \mathrm{~g}$ per aquarium). The feed was placed in a Waring blender at low speed, and zinc $\left(\mathrm{ZnCl}_{2}\right.$ or nano- $\mathrm{ZnO}, 50 \mathrm{~nm}$ diameter from Sigma Chemical Company) was added in $10 \times 100 \mu \mathrm{L}$ volume increments to ensure homogenous distribution in the mixture. Control fish were exposed to aquarium and unspiked feed. The fish were exposed to these conditions at $25^{\circ} \mathrm{C}$ for 21 days under constant aeration $16 \mathrm{~h}$ light and 8 $\mathrm{h}$ dark. Water $\mathrm{pH}$, dissolved oxygen and temperature were monitored daily, and the spawning tiles were checked for egg production (the tiles were replaced with new ones when the egg density was $>50 \%$ of the surface area of the tile). At the end of the exposure period, the fish were anesthetized in a solution of $50 \mathrm{mg} / \mathrm{L} \mathrm{MS}-222$ (Sigma-Aldrich, Ontario, Canada) in accordance with the guidelines of the Animal Care Committee. Fork length and wet body weight, gonad weight and brain weight were recorded. The organs were then mixed with three (different tubes) volumes of homogenization buffer before freezing at $-85^{\circ} \mathrm{C}$. The homogenization buffer consisted of $250 \mathrm{mM}$ sucrose and $25 \mathrm{mM}$ Hepes- $\mathrm{NaOH}$, at $\mathrm{pH} 7.4$, containing $1 \mathrm{mM}$ dithiothreitol and $10 \mu \mathrm{g} / \mathrm{ml}$ apoprotinin (protease inhibitor) and the tissues were homogenized using a Teflon pestle tissue grinder ( 5 passes) on ice. A portion of the homogenate was centrifuged first at $3000 \mathrm{xg}$ for $15 \mathrm{~min}$ at $4^{\circ} \mathrm{C}$, and the resulting supernatant ( $\mathrm{S} 3$ fraction) was centrifuged at $15000 \mathrm{xg}$ for $30 \mathrm{~min}$ at $4^{\circ} \mathrm{C}$. The supernatant ( $\mathrm{S} 15$ fraction), S3 fraction and homogenate were then conserved at $-85^{\circ} \mathrm{C}$ until analysis. The male and female secondary sexual characteristics were also evaluated: ovipositor, banding coloration in males, nuptial tubercles and headsponge appearance. The fish carcasses were set aside for heavy metal analysis by ion plasma mass spectrometry, as described below. 


\section{Metal bioaccumulation}

To determine metal bioaccumulation in fish, tissues were aciddigested with $8 \mathrm{ml}$ of concentrated $\mathrm{HNO}, 1 \mathrm{ml}$ of concentrated $\mathrm{HCl}$, and $2 \mathrm{ml}$ of concentrated $\mathrm{H} 2 \mathrm{O} 2$ (Seastar Baseline). The tissues were then digested during $2 \mathrm{~h}$ at $170^{\circ} \mathrm{C}$ using a microwave digestion system (Ethos EZ, Milestone ScientificInc, ON, Canada). The samples were completed to final volume of $12 \mathrm{ml}$ with deionized water. Total metal concentrations were afterwards determined by XSERIES 2 ICP-MS (Thermo Scientific, USA) and standard solutions of these elements were used for calibration. The data were expressed as $\mu \mathrm{g} / \mathrm{g}$ dry weight and reproductivity was better than $5 \%$. The recovery for these metals was between $90 \%$ and $105 \%$.

\section{Neurotoxicity assessment}

Acetylcholinesterase (AChE) activity was determined using the acetylthiocholine substrate assay [23]. The assay was performed using $3000 \mathrm{x} \mathrm{g}$ supernatant of homogenate in the presence of $0.5 \mathrm{mM}$ acetylthiocholine and Ellman's reagents (5, 5'-Dithiobis (2-nitrobenzoic acid) at pH 7.2 in $100 \mathrm{mM}$ Tris-acetate buffer. The formation of thiocholine in time $\left(0-30 \mathrm{~min}\right.$ at $\left.30^{\circ} \mathrm{C}\right)$ was followed by absorbance at $412 \mathrm{~nm}$ in clear polystyrene microplates (Synergy 4, BioTek Instruments, USA). A calibration curve was prepared using reduced glutathione, and the data was expressed as $\mu$ mole thiols $/ \mathrm{min} /$ $\mathrm{mg}$ protein in the $\mathrm{S} 3$ fraction. Monoamine oxidase (MAO) activity was determined by the coupled dichlorofluorescein-peroxidase activity as described elsewhere [24]. The mitochondria were first isolated by centrifugation of the S3 fraction at $9000 \mathrm{xg}$ for $20 \mathrm{~min}$ at $4^{\circ} \mathrm{C}$, and the mitochondrial pellet was resuspended in the homogenization buffer and referred to as the mitochondrial fraction. The reaction mixture consisted of $100 \mu \mathrm{M}$ tryptamine, $2 \mu \mathrm{M}$ dichlorofluorescein, $100 \mu \mathrm{M}$ aminotriazole (a catalase inhibitor) and $1 \mu \mathrm{g} / \mathrm{mL}$ horseradish peroxidase. The reaction was started by the addition of 50-100 $\mu \mathrm{g} /$ $\mathrm{mL}$ total protein of the mitochondrial fraction and incubated at $30^{\circ} \mathrm{C}$ for $0,30,45$ and $60 \mathrm{~min}$. Fluorescence readings were taken at $485 \mathrm{~nm}$ excitation and $520 \mathrm{~nm}$ emission at each time. Enzymatic activity was expressed as the increase in fluorescence $/ \mathrm{min} / \mathrm{mg}$ protein. Standard solutions of fluorescein were used for optimal instrument adjustments at these wavelengths and calibration.

Dopamine levels in the S15 fraction were determined using a luminescent enzyme-linked immunoassay, as previously described [8]. Briefly, 96-well luminescence plates (Microlite 2, Thermo Fisher Scientific, Ontario, Canada) were coated with $0.5 \mu \mathrm{g}$ of BSAconjugated dopamine (US Biological, Boston, USA) in $50 \mathrm{mM}$ Tris$\mathrm{HCl}, \mathrm{pH} 8.5$ at $4^{\circ} \mathrm{C}$ overnight. The wells were washed with $100 \mu \mathrm{L}$ PBS and $50 \mu \mathrm{L}$ of dopamine standard, and prediluted S15 fraction samples and a blank were added to the wells. One volume of primary antibody (diluted at 1:5000; rabbit polyclonal to dopamine ab888, Abcam, MA, USA) was added. After incubation for $90 \mathrm{~min}$ at room temperature, the wells were washed three times with PBS and incubated with 100 $\mu \mathrm{L}$ of peroxidase-conjugated goat anti-rabbit IgC (1:10 000; StressGen, MI, USA) for 1 hour. Wells were washed three times with PBS, and peroxidase activity was determined using a chemiluminescent substrate (BM Chemiluminescence ELISA Substrate, Roche Diagnostics, QC, Canada). Luminescence readings were taken after 5 min using a luminescence microplate reader (Synergy 4, BioTek Instruments, USA). The data were expressed as $\mu \mathrm{mol}$ dopamine $/ \mathrm{mg}$ protein in the S15 fraction.

Metallothioneins, oxidative stress and genotoxicity

\section{assessments}

Lipid peroxidation (LPO) was determined in brain homogenates using the thiobarbituric acid method [25]. A volume of $50 \mu \mathrm{L}$ of the homogenate was mixed with $200 \mu \mathrm{L}$ of $10 \%$ trichloroacetic acid containing $1 \mathrm{mM} \mathrm{FeSO}_{4}$ and $100 \mu \mathrm{L}$ of $0.7 \%$ thiobarbituric acid and heated at $70-80^{\circ} \mathrm{C}$ for $10 \mathrm{~min}$. The mixture was cooled to room temperature and centrifuged at $10000 \mathrm{x} \mathrm{g}$ for $5 \mathrm{~min}$ to remove any precipitates. A $200 \mu \mathrm{l}$ volume was transferred to a 96-well dark microplate, and fluorescence readings were taken at $520 \mathrm{~nm}$ excitation and $600 \mathrm{~nm}$ emission. Standard solutions of tetramethoxypropane (stabilized form of malonaldehyde) were prepared for calibration on the blank (homogenization buffer). Results were expressed as $\mu$ mole thiobarbituric acid reactants (TBARS)/mg total proteins in the homogenate. DNA damage was determined in brain homogenates using the alkaline precipitation assay [26] which is based on the precipitation of DNA-proteins in potassium dodecyl sulfate. Proteinfree DNA strand breaks were measured in the supernatant by fluorometry. A $25-\mu \mathrm{L}$ sample of the homogenate was mixed with 225 $\mu \mathrm{L}$ detergent solution (2\% SDS containing $10 \mathrm{mM}$ EDTA, $10 \mathrm{mM}$ Tris base and $40 \mathrm{mM} \mathrm{NaOH}$ ) for $1 \mathrm{~min}$, followed by the addition of 250 $\mu \mathrm{L}$ of $0.12 \mathrm{M} \mathrm{KCl}$. The mixture was mixed by inversion, incubated at $60^{\circ} \mathrm{C}$ for $10 \mathrm{~min}$, cooled on ice for $15 \mathrm{~min}$ and centrifuged at $8000 \mathrm{x}$ $\mathrm{g}$ for $10 \mathrm{~min}$. A $50 \mu \mathrm{L}$ volume of the supernatant was mixed with 150 $\mu \mathrm{L}$ of $100 \mu \mathrm{g} / \mathrm{mL}$ Hoescht in $0.1 \mathrm{M}$ Tris-Acetate, $\mathrm{pH} 8.5$, containing 4 $\mathrm{mM}$ sodium cholate and $0.4 \mathrm{M} \mathrm{NaCl}$, and fluorescence readings were taken at $360 \mathrm{~nm}$ excitation and $450 \mathrm{~nm}$ emission. Standard solutions of salmon sperm DNA was prepared for calibration. The data were expressed as $\mu \mathrm{g}$ DNA strand/mg total protein in the homogenate.

Metallothionein (MT) levels were determined using the thiol spectrophotometric assay developed by [27] Viarengo et al. (1997) with some modifications. Since MT is also involved in the sequestration of oxygen radicals in addition to metal binding, a strong reduction step was added before the fractionation steps. Briefly, $100 \mu \mathrm{L}$ of the S15 fraction were treated with $25 \mathrm{mM}$ of Tris(2-carboxyethyl)phosphine for $30 \mathrm{~min}$ to ensure complete reduction [28]. The data were expressed as $\mu \mathrm{g}$ thiols/mg proteins.

\section{Mitochondrial electron transport activity}

Mitochondrial electron transport activity was determined using a dye reduction method, as previously described [29]. The assay is based on the reduction of a tetrazolium dye which was significantly associated with cellular respiration rates $\left(\mathrm{CO}_{2}\right.$ production) in miscellaneous organisms [30]. The crude mitochondrial fraction was first obtained by centrifuging the $\mathrm{S} 3$ fraction at $9000 \mathrm{xg}$ for $20 \mathrm{~min}$ at $4^{\circ} \mathrm{C}$. The pellet was resuspended in $0.1 \mathrm{M}$ Tris- $\mathrm{HCl}, \mathrm{pH} 8.5$, containing $0.1 \mathrm{mM} \mathrm{MgSO}_{4}$, $0.1 \%$ Triton $\mathrm{X}-100$ and $5 \%$ polyvinylpyrrolidone and resuspended by pipette aspiration. $1 \mathrm{mM}$ of $\mathrm{NADH}$ and $0.2 \mathrm{mM} \mathrm{NAPDH}$ were then added to the mitochondrial suspension. The reaction was started by adding $1 \mathrm{mM}$ of p-iodonitrotetrazolium. The reaction was allowed to proceed at $20^{\circ} \mathrm{C}$ for $30 \mathrm{~min}$ and absorbance readings were taken at $520 \mathrm{~nm}$ at 5 -min intervals. The data were expressed as absorbance increase $/ 30 \mathrm{~min} / \mathrm{mg}$ mitochondrial protein content.

\section{Data analysis}

The exposure experiment consisted of 2 males and 4 females per treatment aquarium and the experiment was repeated twice. Tissue biomarkers were analyzed in $\mathrm{N}=4$ males and $\mathrm{N}=4$ females fish using two-way factorial analysis of variance (exposure groups and sex as the main factors) after verifying for homogeneity of variance and normality 
using Levene's test and the Shapiro-Wilk test. Correlation analysis was also performed using the Pearson product moment correlation. To determine the physiological changes induced by exposure to municipal effluents and the two zinc formulations in the feed, a discriminant function analysis and factorial analysis were performed. All statistical tests were performed using Statistica software (version 8). Significance was set at $\alpha=0.05$.

\section{Results}

In fish exposed to the municipal effluents and to each form of $\mathrm{Zn}$, the condition factor (weight/fork length) was not significantly affected by the municipal effluent alone or with $\mathrm{Zn}$ treatments. There was a significant difference between sexes, with males being about 2.2 times larger than females, which is normal for this species. However, there was a significant difference between sexes and a marginal interaction between these two factors $(p=0.08)$. In females, neither municipal effluent nor dietary $\mathrm{Zn}$ treatments were observed to have significant effects. In males, there was no significant change, although the data had more variance, but the difference between males and females was no longer significant for fish fed to nano- $\mathrm{ZnO}$ alone. This small effect was lost however when municipal effluent was present. The gonadosomatic index (GSI) was significantly affected by both effluentdietary $\mathrm{Zn}$ treatments and sex (two-way factorial ANOVA: effluent/Zn forms $>$ sex $>$ effluent/Zn treatments ${ }^{\star}$ sex interaction). In control fish, the GSI in males was significantly higher than in females. In males, the GSI was significantly increased at $20 \%$ effluent, but decreased when the fish were fed nano- $\mathrm{ZnO}$ - or $\mathrm{ZnCl}_{2}$-spiked food, which suggests than exposure to municipal effluent increased the GSI, while dietary $\mathrm{Zn}$ decreased the GSI. In females, the GSI increased with municipal effluent concentration, while exposure to both forms of $\mathrm{Zn}$ in the diet had little effects on the GSI, although $\mathrm{ZnCl}_{2}$ increased the GSI compared to female controls and females exposed to nano- $\mathrm{ZnO}$. A significant interaction between the two factors was also observed in the presence of the municipal effluent and the two forms of dietary $\mathrm{Zn}$, with the GSI of females tending to be higher than that of males. Hence, males and females seem to respond differently to exposure to municipal effluent and forms of Zn. The GSI in males was seemingly more responsive to the decreasing effects of $\mathrm{Zn}$ than to stimulation by the municipal effluent, while in females, the effects of the municipal effluent were more apparent. The brain somatic index (BSI) was significantly affected by sex, exposure treatments with a significant sex ${ }^{*}$ effluent/Zn treatments interaction (factorial two-way ANOVA: sex $>\operatorname{sex}^{*}$ effluent/Zn treatments interaction $>$ effluent/Zn treatments). The BSI in the controls was significantly higher in males than in females. Exposure to the municipal effluent decreased the BSI in males only, while nano- $\mathrm{ZnO}$ alone had effects. Exposure to combined dietary nano- $\mathrm{ZnO}$ and $\mathrm{ZnCl}_{2}$ with the municipal effluent increased the BSI but with less intensity than the municipal effluent alone. Correlation analysis (Table 2) revealed that the condition factor (fish weight/fork length) was not correlated with GSI but was positively correlated with BSI $(\mathrm{r}=0.67 ; \mathrm{p}<0.001)$.

Metal levels in fish carcasses were also determined (Table 3). Tissue Zn levels were significantly affected by sex (factorial ANOVA: sex at $\mathrm{p}<0.001$ ) only. The difference of $\mathrm{Zn}$ in fish tissue tended to be higher in females than in males and this difference increased when dietary $\mathrm{ZnCl}_{2}$ or nano- $\mathrm{ZnO}$ was present with the municipal effluent. However, neither the municipal effluent nor the forms of $\mathrm{Zn}$ in the diet alone influenced the difference in $\mathrm{Zn}$ levels in males and females. Total $\mathrm{Zn}$ levels in fish carcasses were significantly correlated with nickel $((\mathrm{Ni} ; \mathrm{r}=0.28 ; \mathrm{p}<0.01)$ and copper $(\mathrm{r}=0.2 ; \mathrm{p}=0.05)$. The levels of $\mathrm{Ni}$ were similar for both sexes in the controls. However, Ni was significantly increased in females by $20 \%$ municipal effluent, $\mathrm{ZnCl}_{2}$, and 10-20\% municipal effluent in fish fed a $\mathrm{ZnCl}_{2}$-supplemented feed. In males, $\mathrm{Ni}$ was significantly induced at $10 \%$ municipal effluent in fish fed $\mathrm{ZnCl}_{2}$ supplements only. For copper $(\mathrm{Cu})$ in carcasses, both the municipal effluent concentrations/dietary

Table 1. General health status of Fathead Minnows exposed to municipal effluent, $\mathrm{ZnO}$ and dissolved $\mathrm{Zn}$.

\begin{tabular}{|c|c|c|c|c|c|c|}
\hline & Sex & Fork length (mm) & $\begin{array}{l}\text { Fish weight } \\
\text { (g) }\end{array}$ & $\begin{array}{c}\text { Condition } \\
\text { factor }(\mathrm{g} / \mathrm{mm})\end{array}$ & GSI & Brain SI \\
\hline Controls & $\begin{array}{l}\mathrm{M} \\
\mathrm{F}\end{array}$ & $\begin{array}{c}68.3 \pm 1.7 \\
46.25 \pm 2.1^{\mathrm{c}}\end{array}$ & $\begin{array}{l}5.59 \pm 0.68 \\
1.25 \pm 0.13^{\mathrm{c}}\end{array}$ & $\begin{array}{l}0.082 \pm 0.009 \\
0.027 \pm 0.002^{\mathrm{c}}\end{array}$ & $\begin{array}{c}0.09 \pm 0.01 \\
0.022 \pm 0.007^{\mathrm{c}}\end{array}$ & $\begin{array}{c}0.027 \pm 0.006 \\
0.01 \pm 0.001^{\mathrm{c}}\end{array}$ \\
\hline $5 \%$ effluent & $\begin{array}{l}\mathrm{M} \\
\mathrm{F}\end{array}$ & $\begin{array}{c}77 \pm 1 \\
44 \pm 6^{c}\end{array}$ & $\begin{array}{c}8.39 \pm 1.1 \\
1.07 \pm 0.41^{\mathrm{c}}\end{array}$ & $\begin{array}{c}0.11 \pm 0.02 \\
0.02 \pm 0.006^{\mathrm{c}}\end{array}$ & $\begin{array}{l}0.014 \pm 0.007^{\mathrm{a}} \\
0.049 \pm 0.005^{\mathrm{c}}\end{array}$ & $\begin{aligned} 0.007 & \pm 0.001^{\mathrm{a}} \\
0.008 & \pm 0.001\end{aligned}$ \\
\hline $10 \%$ effluent & $\begin{array}{l}\mathrm{M} \\
\mathrm{F}\end{array}$ & $\begin{array}{c}73 \pm 22 \\
53 \pm 15^{\mathrm{c}}\end{array}$ & $\begin{array}{l}7.2 \pm 0.41 \\
3.09 \pm 2.3^{c}\end{array}$ & $\begin{array}{l}0.09 \pm 0.026 \\
0.05 \pm 0.029^{\mathrm{c}}\end{array}$ & $\begin{array}{c}0.007 \pm 0.001^{\mathrm{a}} \\
0.048 \pm 0.04\end{array}$ & $\begin{array}{c}0.009 \pm 5 \mathrm{e}^{-4 \mathrm{a}} \\
0.008 \pm 0.003\end{array}$ \\
\hline $20 \%$ effluent & $\begin{array}{l}\mathrm{M} \\
\mathrm{F}\end{array}$ & $\begin{array}{c}75 \pm 3 \\
45 \pm 3^{c}\end{array}$ & $\begin{array}{l}7.35 \pm 0.27 \\
1.48 \pm 0.24^{\mathrm{c}}\end{array}$ & $\begin{array}{r}0.098 \pm 0.007 \\
0.03 \pm 0.003^{c}\end{array}$ & $\begin{array}{l}0.011 \pm 0.001^{\mathrm{a}} \\
0.013 \pm 0.001\end{array}$ & $\begin{array}{c}0.008 \pm 0.003 \\
0.02 \pm 0.01\end{array}$ \\
\hline $\begin{array}{l}\mathrm{ZnO} \\
(100 \mathrm{ug} / \mathrm{g})\end{array}$ & $\begin{array}{l}\mathrm{M} \\
\mathrm{F}\end{array}$ & $\begin{array}{l}58 \pm 2 \\
50 \pm 1\end{array}$ & $\begin{array}{l}3.41 \pm 0.05 \\
1.54 \pm 0.05\end{array}$ & $\begin{array}{l}0.06 \pm 0.003 \\
0.03 \pm 0.001\end{array}$ & $\begin{array}{c}0.045 \pm 0.005^{\mathrm{a}} \\
0.045 \pm 0.005\end{array}$ & $\begin{array}{c}0.01 \pm 0.001 \\
0.02 \pm 0.01\end{array}$ \\
\hline $\begin{array}{l}\mathrm{ZnCl}_{2} \\
(100 \mathrm{ug} / \mathrm{g})\end{array}$ & $\begin{array}{l}\mathrm{M} \\
\mathrm{F}\end{array}$ & $\begin{array}{l}66.5 \pm 1.5 \\
49.9 \pm 0.5\end{array}$ & $\begin{array}{l}5.16 \pm 0.24 \\
1.5 \pm 0.05^{\mathrm{c}}\end{array}$ & $\begin{array}{c}0.078 \pm 0.005 \\
0.03 \pm 0.001^{\mathrm{c}}\end{array}$ & $\begin{array}{c}0.07 \pm 0.005 \\
0.09 \pm 0.005^{\mathrm{b}}\end{array}$ & $\begin{array}{l}0.02 \pm 0.01 \\
0.04 \pm 0.01\end{array}$ \\
\hline $5 \%$ effluent- $\mathrm{ZnO}$ & $\begin{array}{l}\mathrm{M} \\
\mathrm{F}\end{array}$ & $\begin{array}{c}69 \pm 10 \\
47.5 \pm 1.5^{c}\end{array}$ & $\begin{array}{c}5.44 \pm 2.2 \\
1.43 \pm 0.15^{\mathrm{c}}\end{array}$ & $\begin{array}{c}0.077 \pm 0.02 \\
0.03 \pm 0.002^{\mathrm{c}}\end{array}$ & $\begin{array}{l}0.065 \pm 0.005 \\
0.14 \pm 0.001^{\mathrm{b}, \mathrm{c}}\end{array}$ & $\begin{array}{l}0.015 \pm 0.005 \\
0.045 \pm 0.01^{\mathrm{c}}\end{array}$ \\
\hline $5 \%$ effluent- $\mathrm{ZnCl}_{2}$ & $\begin{array}{l}\mathrm{M} \\
\mathrm{F}\end{array}$ & $\begin{array}{l}69.5 \pm 5.5 \\
49.5 \pm 4.5^{\mathrm{c}}\end{array}$ & $\begin{array}{c}6.13 \pm 1.6 \\
1.45 \pm 0.32^{\mathrm{c}}\end{array}$ & $\begin{array}{c}0.087 \pm 0.016 \\
0.03 \pm 0.004^{\circ}\end{array}$ & $\begin{array}{c}0.08 \pm 0.02 \\
0.11 \pm 0.001^{b}\end{array}$ & $\begin{aligned} 0.045 & \pm 0.005 \\
0.015 & \pm 0.005^{\circ}\end{aligned}$ \\
\hline $10 \%$ effluent- $\mathrm{ZnO}$ & $\begin{array}{l}\mathrm{M} \\
\mathrm{F}\end{array}$ & $\begin{array}{l}80 \pm 1^{\mathrm{a}} \\
46 \pm 4^{\mathrm{c}}\end{array}$ & $\begin{array}{c}7.4 \pm 0.93 \\
1.28 \pm 0.2^{\mathrm{c}}\end{array}$ & $\begin{array}{c}0.092 \pm 0.01 \\
0.028 \pm 0.002^{\mathrm{c}}\end{array}$ & $\begin{array}{c}0.045 \pm 0.01^{\mathrm{a}} \\
0.07 \pm 0.03^{\mathrm{b}}\end{array}$ & $\begin{aligned} 0.05 & \pm 0.01^{\mathrm{a}} \\
0.015 & \pm 0.001^{\mathrm{c}}\end{aligned}$ \\
\hline $10 \%$ effluent- $\mathrm{ZnCl}_{2}$ & $\begin{array}{l}\mathrm{M} \\
\mathrm{F}\end{array}$ & $\begin{aligned} 68.5 & \pm 6.5 \\
50 & \pm 1\end{aligned}$ & $\begin{array}{c}5.0 \pm 0.8 \\
1.45 \pm 0.14^{\mathrm{c}}\end{array}$ & $\begin{array}{c}0.07 \pm 0.005 \\
0.03 \pm 0.002^{\mathrm{c}}\end{array}$ & $\begin{array}{c}0.086 \pm 0.02 \\
0.085 \pm 0.02^{\mathrm{b}}\end{array}$ & $\begin{array}{c}0.035 \pm 0.01 \\
0.0150 .005\end{array}$ \\
\hline $20 \%$ effluent- $\mathrm{ZnO}$ & $\begin{array}{l}\mathrm{M} \\
\mathrm{F}\end{array}$ & $\begin{array}{l}76.5 \pm 7.5^{\mathrm{a}} \\
48.5 \pm 1.5^{\mathrm{c}}\end{array}$ & $\begin{array}{l}6.54 \pm 0.85 \\
1.43 \pm 0.42^{\mathrm{c}}\end{array}$ & $\begin{array}{c}0.085 \pm 0.003 \\
0.03 \pm 0.008^{\mathrm{c}}\end{array}$ & $\begin{array}{c}0.11 \pm 0.02 \\
0.22 \pm 0.02^{\mathrm{b}, \mathrm{c}}\end{array}$ & $\begin{array}{c}0.04 \pm--- \\
0.01 \pm 0.002^{c}\end{array}$ \\
\hline $20 \%$ effluent- $\mathrm{ZnCl}_{2}$ & $\begin{array}{l}\mathrm{M} \\
\mathrm{F}\end{array}$ & $\begin{array}{c}67.5 \pm 3.5 \\
50.5 \pm 4.5^{\mathrm{c}}\end{array}$ & $\begin{array}{l}5.09 \pm 0.72 \\
1.87 \pm 0.34\end{array}$ & $\begin{array}{l}0.075 \pm 0.007 \\
0.037 \pm 0.003^{\mathrm{c}}\end{array}$ & $\begin{array}{c}0.08 \pm 0.03 \\
0.28 \pm 0.003^{\mathrm{b}, \mathrm{c}}\end{array}$ & $\begin{array}{c}0.04 \pm--- \\
0.02 \pm 0.001\end{array}$ \\
\hline
\end{tabular}

a. Significantly different in males (treatment groups).

b. Significantly different in females (treatment groups).

c. Significantly difference between males and females (within a same treatment). 
Table 2. Correlation analysis of the biomarker data.

\begin{tabular}{|c|c|c|c|c|c|c|c|c|c|c|c|c|c|c|c|c|c|c|c|}
\hline & \begin{tabular}{|l|} 
Fork \\
length
\end{tabular} & weight & FC & GSI & $\begin{array}{l}\text { Brain } \\
\text { Index }\end{array}$ & $\begin{array}{l}\text { DNA } \\
\text { brain }\end{array}$ & $\begin{array}{l}\text { LPO } \\
\text { brain }\end{array}$ & $\begin{array}{l}\text { MT } \\
\text { Brain }\end{array}$ & DOP & AChE & MAO & MET & $\mathrm{Fe}$ & Co & $\mathbf{N i}$ & $\mathrm{Cu}$ & Zn & Ag & Cd \\
\hline $\begin{array}{l}\text { Fork } \\
\text { length }\end{array}$ & 1 & $\begin{array}{l}\mathbf{0 . 6 9} \\
p<0.001\end{array}$ & \begin{tabular}{|l|}
0.61 \\
$p<0.001$
\end{tabular} & $\begin{array}{l}-0.05 \\
p>0.1\end{array}$ & \begin{tabular}{|l}
$\mathbf{0 . 7 9}$ \\
$\mathbf{p}<0.001$
\end{tabular} & $\begin{array}{l}0.29 \\
p<0.01\end{array}$ & \begin{tabular}{|l|}
$\mathbf{0 . 6 8}$ \\
$\mathbf{p}<0.001$
\end{tabular} & \begin{tabular}{|l|}
-0.36 \\
$p<0.001$
\end{tabular} & $\begin{array}{l}0.06 \\
\mathrm{p}>0.1\end{array}$ & $\begin{array}{l}0.08 \\
p>0.1\end{array}$ & $\begin{array}{l}0.17 \\
p=0.09\end{array}$ & $\begin{array}{l}0.28 \\
p<0.01\end{array}$ & $\begin{array}{l}-0.10 \\
\mathrm{p}>0.1\end{array}$ & $\begin{array}{l}-0.08 \\
\mathrm{p}>0.1\end{array}$ & $\begin{array}{l}-0.25 \\
p<0.05\end{array}$ & $\begin{array}{l}-0.32 \\
p=0.001\end{array}$ & $\begin{array}{l}-\mathbf{0 . 6 3} \\
\mathbf{p}<0.001\end{array}$ & $\begin{array}{l}-0.09 \\
\mathrm{p}>0.1\end{array}$ & $\begin{array}{l}-0.07 \\
p>0.1\end{array}$ \\
\hline weight & & 1 & $\begin{array}{l}0.98 \\
\mathrm{p}<0.001\end{array}$ & $\begin{array}{l}-0.06 \\
p>0.1\end{array}$ & \begin{tabular}{|l|}
$\mathbf{0 . 7 8}$ \\
$\mathbf{p}>\mathbf{0 . 0 0 1}$
\end{tabular} & $\begin{array}{l}0.17 \\
p>0.1\end{array}$ & \begin{tabular}{|l|}
$\mathbf{0 . 3 7}$ \\
$\mathbf{p}<\mathbf{0 . 0 0 1}$
\end{tabular} & \begin{tabular}{|l|}
-0.34 \\
$p=0.001$
\end{tabular} & $\begin{array}{l}0.1 \\
\mathrm{p}>0.1\end{array}$ & $\begin{array}{l}-0.08 \\
p>0.1\end{array}$ & $\begin{array}{l}0.05 \\
p>0.1\end{array}$ & $\begin{array}{l}0.12 \\
\mathrm{p}>0.1\end{array}$ & $\begin{array}{l}-0.07 \\
p>0.1\end{array}$ & $\begin{array}{l}-0.10 \\
p>0.1\end{array}$ & $\begin{array}{l}-0.24 \\
p<0.01\end{array}$ & $\begin{array}{l}-0.23 \\
p<0.05\end{array}$ & $\begin{array}{l}-\mathbf{0 . 3 9} \\
\mathbf{p}<0.001\end{array}$ & $\begin{array}{l}-0.12 \\
\mathrm{p}>0.1\end{array}$ & $\begin{array}{l}-0.05 \\
\mathrm{p}>0.1\end{array}$ \\
\hline FC & & & 1 & $\begin{array}{l}-0.09 \\
p>0.1\end{array}$ & \begin{tabular}{|l}
$\mathbf{0 . 6 7}$ \\
$\mathbf{p}<0.001$
\end{tabular} & $\begin{array}{l}0.18 \\
\mathrm{p}=0.08\end{array}$ & \begin{tabular}{|l|}
0.29 \\
$p<0.01$
\end{tabular} & \begin{tabular}{|l|l}
-0.33 \\
$p=0.001$
\end{tabular} & $\begin{array}{l}0.07 \\
p>0.1\end{array}$ & $\begin{array}{l}-0.11 \\
p>0.1\end{array}$ & $\begin{array}{l}-0.05 \\
\mathrm{p}>0.1\end{array}$ & $\begin{array}{l}0.10 \\
\mathrm{p}>0.1\end{array}$ & $\begin{array}{l}-0.05 \\
p>0.1\end{array}$ & $\begin{array}{l}-0.1 \\
\mathrm{p}>0.1\end{array}$ & $\begin{array}{l}-0.21 \\
p<0.05\end{array}$ & $\begin{array}{l}-0.20 \\
p=0.05\end{array}$ & \begin{tabular}{|l|}
$-\mathbf{0 . 3 7}$ \\
$\mathbf{p}<0.001$
\end{tabular} & $\begin{array}{l}-0.12 \\
\mathrm{p}>0.1\end{array}$ & $\begin{array}{l}-0.02 \\
p>0.1\end{array}$ \\
\hline GSI & & & & 1 & $\begin{array}{l}-0.07 \\
p>0.1\end{array}$ & $\begin{array}{l}0.11 \\
p>0.1\end{array}$ & $\begin{array}{l}-0.08 \\
p>0.1\end{array}$ & $\begin{array}{l}-0.19 \\
p=0.06\end{array}$ & $\begin{array}{l}0.35 \\
p<0.001\end{array}$ & $\begin{array}{l}-0.04 \\
\mathrm{p}>0.1\end{array}$ & $\begin{array}{l}0.04 \\
p>0.1\end{array}$ & $\begin{array}{l}0.09 \\
p>0.1\end{array}$ & \begin{tabular}{|l|}
.49 \\
$p<0.001$
\end{tabular} & $\begin{array}{l}0.61 \\
p<0.001\end{array}$ & $\begin{array}{l}0.47 \\
p<0.01\end{array}$ & $\begin{array}{l}\mathbf{0 . 5 5} \\
\mathbf{p}<0.001\end{array}$ & $\begin{array}{l}0.15 \\
p>0.1\end{array}$ & $\begin{array}{l}\mathbf{0 . 6 0} \\
\mathbf{p}<0.001\end{array}$ & $\begin{array}{l}-0.30 \\
p<0.01\end{array}$ \\
\hline $\begin{array}{l}\text { Brain } \\
\text { Index }\end{array}$ & & & & & 1 & $\begin{array}{l}0.17 \\
\mathrm{p}=0.09\end{array}$ & $\begin{array}{l}\mathbf{0 . 5 5} \\
\mathbf{p}<0.001\end{array}$ & $\begin{array}{l}-\mathbf{- 0 . 3 6} \\
\mathbf{p}<0.001\end{array}$ & $\begin{array}{l}0.04 \\
p>0.1\end{array}$ & $\begin{array}{l}0.03 \\
\mathrm{p}>0.1\end{array}$ & $\begin{array}{l}0.18 \\
p=0.07\end{array}$ & $\begin{array}{l}0.15 \\
\mathrm{p}>0.1\end{array}$ & \begin{tabular}{|l|}
-0.20 \\
$p<0.05$
\end{tabular} & $\begin{array}{l}-0.18 \\
\mathrm{p}=0.07\end{array}$ & $\begin{array}{l}-0.20 \\
p=0.06\end{array}$ & $\begin{array}{l}-\mathbf{- 0 . 3 0} \\
\mathbf{p}<0.01\end{array}$ & $\begin{array}{l}-\mathbf{0 . 4 0} \\
\mathbf{p}<0.001\end{array}$ & $\begin{array}{l}-0.16 \\
p>0.1\end{array}$ & $\begin{array}{l}-0.15 \\
p>0.1\end{array}$ \\
\hline $\begin{array}{l}\text { DNA } \\
\text { brain }\end{array}$ & & & & & & 1 & $\begin{array}{l}0.40 \\
p<0.001\end{array}$ & $\begin{array}{l}0.09 \\
\mathrm{p}>0.1\end{array}$ & $\begin{array}{l}-0.10 \\
p>0.1\end{array}$ & $\begin{array}{l}0.25 \\
p<0.05\end{array}$ & $\begin{array}{l}0.10 \\
\mathrm{p}>0.1\end{array}$ & $\begin{array}{l}0.36 \\
p<0.001\end{array}$ & $\begin{array}{l}-0.11 \\
p>0.1\end{array}$ & $\begin{array}{l}-0.11 \\
p>0.1\end{array}$ & $\begin{array}{l}-0.15 \\
p>0.1\end{array}$ & $\begin{array}{l}-0.30 \\
p<0.01\end{array}$ & $\begin{array}{l}-0.29 \\
p<0.01\end{array}$ & $\begin{array}{l}-0.07 \\
p>0.1\end{array}$ & $\begin{array}{l}-0.16 \\
p>0.1\end{array}$ \\
\hline $\begin{array}{l}\text { LPO } \\
\text { brain }\end{array}$ & & & & & & & 1 & $\begin{array}{l}-0.30 \\
p<0.01\end{array}$ & $\begin{array}{l}-0.05 \\
p>0.1\end{array}$ & $\begin{array}{l}0.37 \\
p<0.001\end{array}$ & $\begin{array}{l}0.36 \\
p<0.001\end{array}$ & $\begin{array}{l}0.33 \\
p=0.001\end{array}$ & $\begin{array}{l}-0.17 \\
p=0.1\end{array}$ & $\begin{array}{l}-0.17 \\
p=0.08\end{array}$ & $\begin{array}{l}-0.31 \\
p<0.01\end{array}$ & $\begin{array}{l}-0.35 \\
p<0.001\end{array}$ & $\begin{array}{l}-0.44 \\
p<0.001\end{array}$ & $\begin{array}{l}-0.16 \\
p>0.1\end{array}$ & $\begin{array}{l}-0.25 \\
p=0.01\end{array}$ \\
\hline $\begin{array}{l}\text { MT } \\
\text { Brain }\end{array}$ & & & & & & & & 1 & $\begin{array}{l}-0.04 \\
p>0.1\end{array}$ & $\begin{array}{l}0.20 \\
\mathrm{p}=0.05\end{array}$ & $\begin{array}{l}0.29 \\
p<0.01\end{array}$ & \begin{tabular}{|l|}
$\mathbf{0 . 3 1}$ \\
$\mathbf{p}<0.01$
\end{tabular} & \begin{tabular}{|l|}
-0.28 \\
$p<0.01$
\end{tabular} & \begin{tabular}{|l|}
$\mathbf{0 . 2 8}$ \\
$\mathrm{p}<0.01$
\end{tabular} & $\begin{array}{l}-0.17 \\
p=0.1\end{array}$ & $\begin{array}{l}-0.21 \\
p<0.05\end{array}$ & $\begin{array}{l}0.03 \\
\mathrm{p}>0.1\end{array}$ & $\begin{array}{l}-0.25 \\
p<0.05\end{array}$ & $\begin{array}{l}-0.21 \\
p<0.05\end{array}$ \\
\hline DOP & & & & & & & & & 1 & $\begin{array}{l}0.06 \\
p>0.1\end{array}$ & $\begin{array}{l}\mathbf{0 . 3 1} \\
\mathbf{p}<0.001\end{array}$ & $\begin{array}{l}-0.32 \\
p<0.001\end{array}$ & $\begin{array}{l}\mathbf{0 . 3 7} \\
\mathbf{p}<\mathbf{0 . 0 0 1}\end{array}$ & $\begin{array}{l}0.45 \\
p<0.001\end{array}$ & $\begin{array}{l}0.40 \\
p<0.001\end{array}$ & $\begin{array}{l}\mathbf{0 . 3 3} \\
\mathrm{p}=\mathbf{0 . 0 0 1}\end{array}$ & $\begin{array}{l}-0.1 \\
p>0.1\end{array}$ & $\begin{array}{l}0.46 \\
p<0.001\end{array}$ & $\begin{array}{l}0.25 \\
p=0.01\end{array}$ \\
\hline AChE & & & & & & & & & & 1 & \begin{tabular}{|l|}
0.62 \\
$p<0.001$
\end{tabular} & $\begin{array}{l}0.17 \\
p=0.1\end{array}$ & $\begin{array}{l}-0.15 \\
\mathrm{p}>0.1\end{array}$ & $\begin{array}{l}-0.11 \\
p>0.1\end{array}$ & $\begin{array}{l}-0.16 \\
p>0.1\end{array}$ & $\begin{array}{l}0.01 \\
p>0.1\end{array}$ & $\begin{array}{l}0.06 \\
\mathrm{p}>0.1\end{array}$ & $\begin{array}{l}-0.02 \\
p>0.1\end{array}$ & $\begin{array}{l}0.03 \\
p>0.1\end{array}$ \\
\hline MAO & & & & & & & & & & & 1 & $\begin{array}{l}0.003 \\
p>0.1\end{array}$ & $\begin{array}{l}-0.24 \\
p<0.05\end{array}$ & $\begin{array}{l}-0.17 \\
p=0.1\end{array}$ & $\begin{array}{l}-0.19 \\
p=0.08\end{array}$ & $\begin{array}{l}-0.23 \\
p<0.05\end{array}$ & $\begin{array}{l}-0.04 \\
p>0.1\end{array}$ & $\begin{array}{l}-0.16 \\
p>0.1\end{array}$ & $\begin{array}{l}-0.32 \\
p=0.001\end{array}$ \\
\hline $\begin{array}{l}\text { MET } \\
\text { Brain }\end{array}$ & & & & & & & & & & & & 1 & $\begin{array}{l}-0.05 \\
p>0.1\end{array}$ & $\begin{array}{l}-0.07 \\
p>0.1\end{array}$ & $\begin{array}{l}-0.01 \\
p>0.1\end{array}$ & $\begin{array}{l}-\mathbf{0 . 2 1} \\
\mathrm{p}<0.05\end{array}$ & $\begin{array}{l}-0.09 \\
p>0.1\end{array}$ & $\begin{array}{l}-0.04 \\
p>0.1\end{array}$ & $\begin{array}{l}-0.02 \\
p>0.1\end{array}$ \\
\hline $\mathrm{Fe}$ & & & & & & & & & & & & & 1 & \begin{tabular}{|l|}
0.96 \\
$p<0.001$
\end{tabular} & $\begin{array}{l}\mathbf{0 . 7 5} \\
\mathbf{p}<0.001\end{array}$ & \begin{tabular}{|l|}
0.66 \\
$p<0.001$
\end{tabular} & $\begin{array}{l}0.13 \\
\mathrm{p}>0.1\end{array}$ & $\begin{array}{l}\mathbf{0 . 9 3} \\
\mathbf{p}<0.001\end{array}$ & $\begin{array}{l}0.73 \\
p<0.001\end{array}$ \\
\hline Co & & & & & & & & & & & & & & 1 & $\begin{array}{l}\mathbf{0 . 7 6} \\
\mathbf{p}<0.001\end{array}$ & $\begin{array}{l}\mathbf{0 . 7 3} \\
\mathrm{p}<0.001\end{array}$ & $\begin{array}{l}0.11 \\
\mathrm{p}>0.1\end{array}$ & $\begin{array}{l}\mathbf{0 . 9 7} \\
\mathbf{p}<0.001\end{array}$ & $\begin{array}{l}0.71 \\
p<0.001\end{array}$ \\
\hline $\mathbf{N i}$ & & & & & & & & & & & & & & & 1 & $\begin{array}{l}0.64 \\
p<0.001\end{array}$ & $\begin{array}{l}0.28 \\
p<0.01\end{array}$ & $\begin{array}{l}\mathbf{0 . 7 4} \\
\mathbf{p}<0.001\end{array}$ & $\begin{array}{l}0.66 \\
p<0.001\end{array}$ \\
\hline $\mathrm{Cu}$ & & & & & & & & & & & & & & & & 1 & $\begin{array}{l}0.2 \\
p=0.05\end{array}$ & $\begin{array}{l}0.72 \\
p<0.001\end{array}$ & $\begin{array}{l}0.63 \\
p<0.001\end{array}$ \\
\hline $\mathbf{Z n}$ & & & & & & & & & & & & & & & & & 1 & $\begin{array}{l}0.1 \\
p>0.1\end{array}$ & $\begin{array}{l}0.17 \\
p=0.08\end{array}$ \\
\hline Ag & & & & & & & & & & & & & & & & & & 1 & $\begin{array}{l}0.73 \\
p<0.001\end{array}$ \\
\hline
\end{tabular}

Table 3. Metal levels in fish carcass.

\begin{tabular}{|c|c|c|c|c|c|c|c|}
\hline $\begin{array}{l}\text { Exposure } \\
\text { conditions }\end{array}$ & $\begin{array}{c}\text { Fe } \\
\mu \mathrm{g} / \mathrm{g} \text { dry weight }\end{array}$ & $\begin{array}{c}\text { Co } \\
\text { ng/g dry weight }\end{array}$ & $\begin{array}{c}\mathrm{Ni} \\
\text { ng/g dry weight }\end{array}$ & $\begin{array}{c}\mathrm{Cu} \\
\mu \mathrm{g} / \mathrm{g} \text { dry weight }\end{array}$ & $\begin{array}{c}\mathrm{Zn} \\
\mu \mathrm{g} / \mathrm{g} \text { dry weight }\end{array}$ & $\begin{array}{c}\text { Ag } \\
\text { ng/g dry weight }\end{array}$ & $\begin{array}{c}\text { Cd } \\
\text { ng/g dry weight }\end{array}$ \\
\hline Controls & $\begin{array}{c}\text { M: } 12.7 \pm 4 \\
\text { F: } 10.7 \pm 1\end{array}$ & $\begin{array}{c}\text { M: } 30 \pm 10 \\
\text { F: } 5 \pm 0.5\end{array}$ & $\begin{array}{c}\text { M: } 19 \pm 4 \\
\text { F: } 27 \pm 6\end{array}$ & $\begin{array}{c}\text { M: } 1.2 \pm 0.08 \\
\text { F: } 1.2 \pm 0.1\end{array}$ & $\begin{array}{c}\text { M: } 38 \pm 6 \\
\text { F: } 53 \pm 3\end{array}$ & $\begin{array}{l}\text { M: } 0.4 \pm 0.01 \\
\text { F: } 0.2 \pm 0.001\end{array}$ & $\begin{array}{l}\text { M: } 10 \pm 3 \\
\text { F: } 6 \pm 0.5^{c}\end{array}$ \\
\hline Effluent $5 \%$ & $\begin{array}{c}\text { M: nd } \\
\text { F: } 6.1 \pm 1\end{array}$ & $\begin{array}{c}\mathrm{M}: 2 \pm 0.5 \\
\mathrm{~F}: 7 \pm 1\end{array}$ & $\begin{array}{c}\text { M: nd } \\
\text { F: } 17 \pm 0.1\end{array}$ & $\begin{array}{c}\text { M: nd } \\
\text { F: } 1.8 \pm 0.7\end{array}$ & $\begin{array}{l}\mathrm{M}: 25 \pm 1 \\
\mathrm{~F}: 46 \pm 10\end{array}$ & $\begin{array}{c}\text { M: nd } \\
\text { F: } 1 \pm 0.03\end{array}$ & $\begin{array}{l}\text { M: nd } \\
\text { F: } 6 \pm 2^{\mathrm{c}}\end{array}$ \\
\hline Effluent $10 \%$ & $\begin{array}{l}\text { M: } 8.2 \pm 2 \\
\text { F: } 18 \pm 3^{b, c}\end{array}$ & $\begin{array}{c}\mathrm{M}: 9 \pm 2 \\
\mathrm{~F}: 81 \pm 30^{\mathrm{b}}\end{array}$ & $\begin{array}{l}\text { M: } 11 \pm 0.5 \\
\text { F: } 30 \pm 0.8\end{array}$ & $\begin{array}{c}\text { M: } 0.98 \pm 0.3 \\
\text { F: } 2.1 \pm 0.1^{\mathrm{c}}\end{array}$ & $\begin{array}{l}\mathrm{M}: 42 \pm 7 \\
\mathrm{~F}: 59 \pm 20\end{array}$ & $\begin{array}{c}\text { M: } 1.2 \pm 0.01 \\
\text { F: } 3 \pm 1^{b}\end{array}$ & $\begin{array}{c}\text { M: } 4.5 \pm 0.1^{\mathrm{a}} \\
\mathrm{F}: 8 \pm 0.2\end{array}$ \\
\hline Effluent $20 \%$ & $\begin{array}{c}\text { M: } 12 \pm 4 \\
\text { F: } 20 \pm 2^{b, c}\end{array}$ & $\begin{array}{l}\text { M: } 73 \pm 20^{\mathrm{a}} \\
\mathrm{F}: 136 \pm 24^{\mathrm{b}}\end{array}$ & $\begin{array}{c}\text { M: } 20 \pm 2 \\
F: 75 \pm 5^{\mathrm{b}, \mathrm{c}}\end{array}$ & $\begin{array}{l}\text { M: } 1.85 \pm 0.4 \\
\text { F: } 4.4 \pm 0.8^{b, c}\end{array}$ & $\begin{array}{c}\mathrm{M}: 28 \pm 1 \\
\mathrm{~F}: 59 \pm 15^{\mathrm{c}}\end{array}$ & $\begin{array}{c}\text { M: } 3.4 \pm 0.1^{\mathrm{a}} \\
\mathrm{F}: 7 \pm 1^{\mathrm{b}, \mathrm{c}}\end{array}$ & $\begin{array}{c}\text { M: } 5.5 \pm 2^{\mathrm{a}} \\
\mathrm{F}: 8 \pm 0.1\end{array}$ \\
\hline $\mathrm{ZnO}$ & $\begin{array}{c}\text { M: } 8.4 \pm 1 \\
\text { F: } 8.5 \pm 0.5\end{array}$ & $\begin{array}{l}\text { M: } 6 \pm 1 \\
\text { F:5 } 5 \pm 0.5\end{array}$ & $\begin{array}{l}\text { M: } 13 \pm 2 \\
\text { F: } 32 \pm 10\end{array}$ & $\begin{array}{l}\text { M: } 1.2 \pm 0.01 \\
\text { F: } 1.25 \pm 0.15\end{array}$ & $\begin{array}{c}\mathrm{M}: 34 \pm 0.3 \\
\mathrm{~F}: 52 \pm 8\end{array}$ & $\begin{array}{l}\text { M: } 0.8 \pm 0.2 \\
\text { F: } 0.4 \pm 0.2\end{array}$ & $\begin{array}{c}\text { M:5.5 } \pm 0.1^{\mathrm{a}} \\
\mathrm{F}: 7 \pm 1\end{array}$ \\
\hline $\mathrm{ZnCl}_{2}$ & $\begin{array}{l}\text { M: } 9 \pm 0.1 \\
\text { F: } 9.3 \pm 1\end{array}$ & $\begin{array}{l}\text { M: } 4 \pm 0.4 \\
\text { F:3.4 }\end{array}$ & $\begin{array}{c}\text { M: } 12 \pm 2 \\
\text { F: } 60 \pm 20^{b, c}\end{array}$ & $\begin{array}{c}\mathrm{M}: 1.3 \pm 0.25 \\
\mathrm{~F}: 1.2 \pm 0.15\end{array}$ & $\begin{array}{c}\text { M: } 24 \pm 2 \\
F: 46 \pm 1\end{array}$ & $\begin{array}{l}\text { M: } 0.6 \pm 0.1 \\
\text { F: } 0.3 \pm 0.1\end{array}$ & $\begin{array}{l}\text { M: } 8.4 \pm 1 \\
\text { F: } 7 \pm 0.5\end{array}$ \\
\hline Effluent $5 \%-\mathrm{ZnO}$ & $\begin{array}{c}\text { M: } 10 \pm 0.05 \\
F: 10 \pm 0.05\end{array}$ & $\begin{array}{c}\text { M: } 7.6 \pm 0.2 \\
\text { F: } 7.6 \pm 0.2\end{array}$ & $\begin{array}{l}\text { M: } 18 \pm 3 \\
\text { F: } 18 \pm 3\end{array}$ & $\begin{array}{c}\text { M: } 1.3 \pm 0.02 \\
\text { F: } 1.3 \pm 0.2\end{array}$ & $\begin{array}{c}\mathrm{M}: 38 \pm 3 \\
\mathrm{~F}: 38 \pm 5\end{array}$ & $\begin{array}{l}\text { M: } 0.9 \pm 0.2 \\
\text { F: } 0.9 \pm 0.1\end{array}$ & $\begin{array}{c}\text { M: } 6 \pm 0.2 \\
F: 6 \pm 0.2\end{array}$ \\
\hline Effluent $10 \%$ - $\mathrm{ZnO}$ & $\begin{array}{c}\text { M: } 10.6 \pm 0.5 \\
\text { F: } 8.6 \pm 0.5\end{array}$ & $\begin{array}{c}\text { M: } 6.5 \pm 0.1^{\mathrm{a}} \\
\text { F: } 6.6 \pm 1\end{array}$ & $\begin{array}{c}\text { M: } 11 \pm 0.5 \\
\text { F: } 11 \pm 3\end{array}$ & $\begin{array}{c}\text { M: } 1.5 \pm 0.1 \\
F: 1.9 \pm 0.8\end{array}$ & $\begin{array}{c}\text { M: } 40 \pm 7 \\
\text { F: } 65 \pm 10^{c}\end{array}$ & $\begin{array}{l}\mathrm{M}: 1 \pm 0.05 \\
\mathrm{~F}: 0.7 \pm 0.2\end{array}$ & $\begin{array}{c}\text { M: } 7.7 \pm 0.4 \\
\text { F: } 7.5 \pm 1\end{array}$ \\
\hline Effluent $20 \%-\mathrm{ZnO}$ & $\begin{array}{l}\text { M: } 10.6 \pm 0.1 \\
\text { F: } 10.5 \pm 0.1\end{array}$ & $\begin{array}{l}\text { M: } 4 \pm 0.3^{\mathrm{a}} \\
\mathrm{F}: 17 \pm 2^{\mathrm{b}}\end{array}$ & $\begin{array}{l}\text { M: } 8 \pm 0.5 \\
\text { F: } 25 \pm 4^{c}\end{array}$ & $\begin{array}{l}\text { M: } 0.7 \pm 0.03 \\
\text { F: } 2.9 \pm 0.6^{b, c}\end{array}$ & $\begin{array}{l}\mathrm{M}: 38 \pm 6 \\
\mathrm{~F}: 55 \pm 10\end{array}$ & $\begin{array}{c}\text { M: } 0.1 \pm 0.03 \\
\text { F: } 0.8 \pm 0.05\end{array}$ & $\begin{array}{c}\mathrm{M}: 4.8 \pm 0.1^{\mathrm{a}} \\
\mathrm{F}: 8.4 \pm 1\end{array}$ \\
\hline Effluent $5 \%-\mathrm{ZnCl}_{2}$ & $\begin{array}{c}\text { M: } 10.7 \pm 2^{\mathrm{a}} \\
\text { F: } 11 \pm 0.8\end{array}$ & $\begin{array}{l}\text { M: } 9 \pm 2^{\mathrm{a}} \\
\mathrm{F}: 15 \pm 2^{\mathrm{b}}\end{array}$ & $\begin{array}{l}\text { M: } 26 \pm 10^{\mathrm{a}} \\
\mathrm{F}: 13 \pm 0.5^{\mathrm{c}}\end{array}$ & $\begin{array}{c}\text { M: } 1.2 \pm 0.1 \\
\text { F: } 1.7 \pm 0.2\end{array}$ & $\begin{array}{c}\text { M: } 30 \pm 4 \\
\text { F: } 67 \pm 10^{c}\end{array}$ & $\begin{array}{c}\text { M: } 0.6 \pm 0.1 \\
F: 0.6 \pm 0.2\end{array}$ & $\begin{array}{c}\text { M: } 5 \pm 0.4^{\mathrm{a}} \\
\mathrm{F}: 5 \pm 0.3\end{array}$ \\
\hline Effluent $10 \%-\mathrm{ZnCl}_{2}$ & $\begin{array}{l}\text { M: } 23 \pm 0.4^{\mathrm{a}} \\
\mathrm{F}: 61 \pm 4^{\mathrm{b}, \mathrm{c}}\end{array}$ & $\begin{array}{l}\text { M: } 157 \pm 8 \\
\text { F: } 442 \pm 30\end{array}$ & $\begin{array}{l}\text { M: } 68 \pm 20^{\mathrm{a}} \\
\mathrm{F}: 122 \pm 2^{\mathrm{b}}\end{array}$ & $\begin{array}{c}\text { M: } 2.4 \pm 0.1^{\mathrm{a}} \\
\mathrm{F}: 4.9 \pm 0.08 \mathrm{~b}, \mathrm{c}\end{array}$ & $\begin{array}{l}\text { M: } 29 \pm 2 \\
\text { F: } 68 \pm 7^{c}\end{array}$ & $\begin{array}{l}\mathrm{M}: 7 \pm 0.1^{\mathrm{a}} \\
\mathrm{F}: 24 \pm 3^{\mathrm{b}, \mathrm{c}}\end{array}$ & $\begin{array}{l}\text { M: } 13 \pm 0.1 \\
\text { F: } 25 \pm 0.5^{b, c}\end{array}$ \\
\hline Effluent $20 \%-\mathrm{ZnCl}_{2}$ & $\begin{array}{c}\text { M: } 14.5 \pm 3^{\mathrm{a}} \\
\text { F: } 18 \pm 1^{\mathrm{b}}\end{array}$ & $\begin{array}{l}\text { M: } 73 \pm 25 \\
\text { F: } 135 \pm 20\end{array}$ & $\begin{array}{l}\text { M: } 18 \pm 3 \\
F: 58 \pm 1^{\mathrm{b}}\end{array}$ & $\begin{array}{c}\mathrm{M}: 2 \pm 0.1^{\mathrm{a}} \\
\mathrm{F}: 4.4 \pm 0.5^{\mathrm{b} . \mathrm{c}}\end{array}$ & $\begin{array}{l}\text { M: } 25 \pm 3 \\
\text { F: } 53 \pm 9^{c}\end{array}$ & $\begin{array}{c}\text { M: } 3.5 \pm 1^{\mathrm{a}} \\
\text { F: } 7.3 \pm 0.5^{\mathrm{bc}}\end{array}$ & $\begin{array}{l}\mathrm{M}: 8 \pm 1 \\
\mathrm{~F}: 10 \pm 1\end{array}$ \\
\hline
\end{tabular}

a. significantly different in males; b. significantly different in females; c. significantly different between males and females in the same treatment group. Nd: not determined 
forms of $\mathrm{Zn}$ and sex were significant (sex>exposure treatments $>\operatorname{sex}^{*}$ effluent/Zn treatments interaction). Cu levels were similar in males and females in the control fish. In females exposed to the municipal effluent, tissue $\mathrm{Cu}$ levels were significantly higher at a $20 \%$ concentration, with

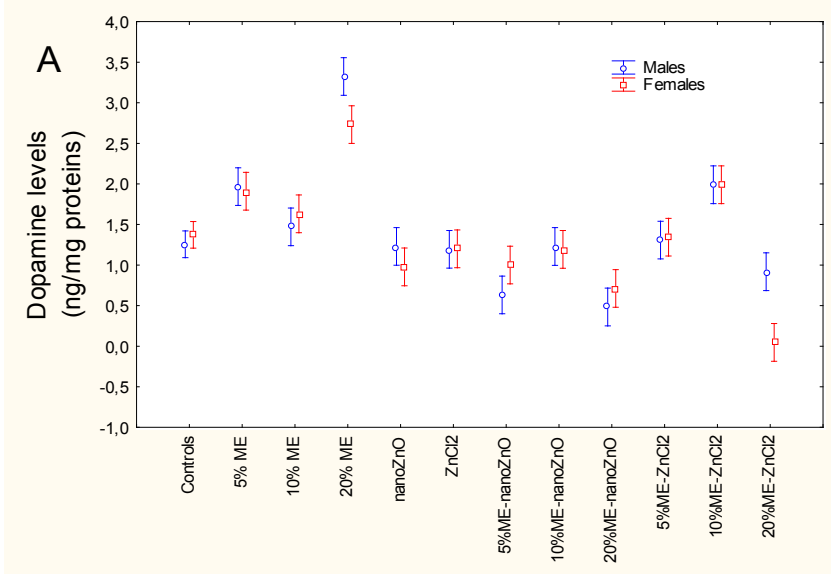

Exposure treatments
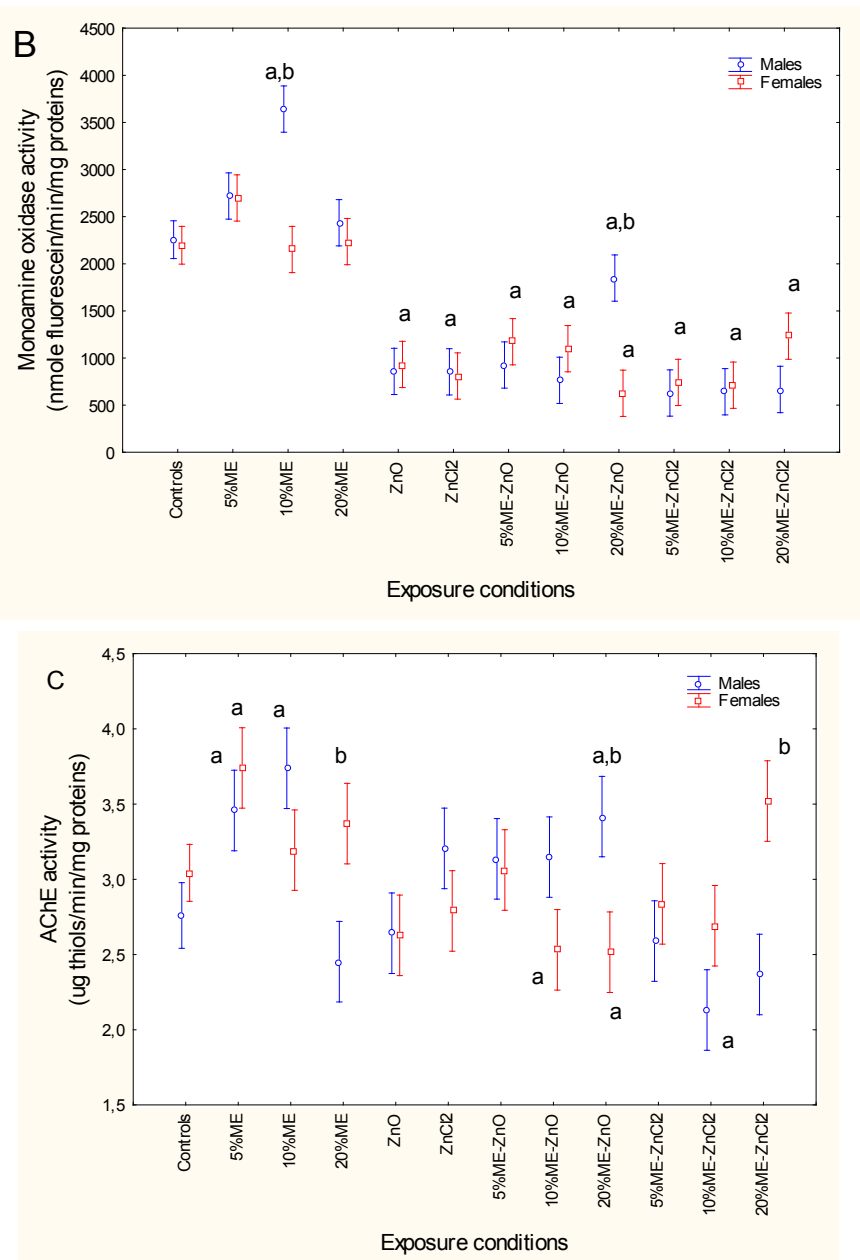

Figure 1. Neurological effects of fathead minnows exposed to $\mathrm{ME}$, nano- $\mathrm{ZnO}$ and $\mathrm{ZnCl}_{2}$. FHM were exposed to the ME, nano- $\mathrm{ZnO}$ and $\mathrm{ZnCl}_{2}$ alone and in combination. The levels of brain dopamine (A), mono-amine oxidase (MOA) activity (B) and acetylcholinesterase $(\mathrm{AChE})$ activity $(\mathrm{C})$ are reported. The letter a indicates significant from controls and $\mathrm{b}$ significant between males-females at a given treatment condition.
nano-Zn-spiked feed, $10 \%$ and $20 \%$ concentrations $\mathrm{ZnCl}_{2}$-spiked diet. In males, $\mathrm{Cu}$ levels were increased only at $10 \%$ effluent concentration with fish fed a $\mathrm{ZnCl}_{2}$-supplemented feed.

Brain dopamine levels were determined in fathead minnows exposed to municipal effluent and dietary forms of $\mathrm{Zn}$ (Figure 1A). FHM were exposed to the $\mathrm{ME}$, nano- $\mathrm{ZnO}$ and $\mathrm{ZnCl}_{2}$ alone and in combination. The levels of brain dopamine (A), mono-amine oxidase (MOA) activity (B) and acetylcholinesterase (AChE) activity (C) are reported. The letter a indicates significant from controls and $b$ significant between males-females at a given treatment condition.

Factorial analysis of variance revealed that only the exposure concentrations were significant. Brain dopamine levels were increased after exposure to $20 \%$ municipal effluent, while exposure to either nano- $\mathrm{ZnO}$ or $\mathrm{ZnCl}_{2}$ alone had no effects. Combined exposure to dietary forms of $\mathrm{Zn}$ and the highest concentration of municipal effluent (20\%) decreased dopamine levels, while exposure to $10 \%$ effluent increased dopamine levels in fish fed a $\mathrm{ZnCl}_{2}$-supplemented feed. Correlation analysis revealed that dopamine was significantly correlated with Co $(r=0.29 ; \mathrm{p}<0.05)$, Ni $(r=0.32 ; \mathrm{p}<0.05)$ and $\mathrm{Ag}(\mathrm{r}=0.31 ; \mathrm{p}<0.05)$. $\mathrm{MAO}$ activity was also determined in brain mitochondria (Figure 1B). Factorial analysis of variance revealed a significant effluent $/ \mathrm{Zn}$ treatments ${ }^{\star}$ sex interaction. In control fish, MOA activity was similar between males and females. Exposure to the municipal effluent led to significant increases in MAO activity, while exposure to either forms of $\mathrm{Zn}$ significantly reduced MAO activity (at 33\% of the controls). Co-exposure to the municipal effluent and either dietary nano- $\mathrm{ZnO}$ or $\mathrm{ZnCl}_{2}$ did not restore MOA activity. Males were more sensitive to exposure to municipal effluent and were less sensitive to dietary nano- $\mathrm{ZnO}$ than to $\mathrm{ZnCl}_{2}$ in the presence of municipal effluent. Correlation analysis revealed that $\mathrm{MAO}$ activity was significantly correlated with dopamine $(r=0.30 ; p<0.05), \mathrm{Fe}(\mathrm{r}=-0.24 ; \mathrm{p}<0.05), \mathrm{Cu}$ $(\mathrm{r}=-0.23 ; \mathrm{p}<0.05)$ and $\mathrm{Cd}(\mathrm{r}=-0.32 ; \mathrm{p}=0.001)$. AChE activity was also determined in brain tissue as a general marker of neural activity (Figure 1C). As with MAO activity, factorial analysis of variance revealed that AChE activity was significantly influenced by a gender-exposure concentration interaction. AChE activity was significantly induced at low concentrations of municipal effluent (5 and 10\%). Exposure to either dietary nano- $\mathrm{ZnO}$ or $\mathrm{ZnCl}_{2}$ did not influence $\mathrm{AChE}$ activity. The combination of municipal effluent and dietary nano- $\mathrm{ZnO}$ produced no change in AChE activity. However, the difference in AChE activity between males and females was significant in fish exposed to $20 \%$ municipal effluent and fed to either forms of $\mathrm{Zn}$. Correlation analysis revealed that $\mathrm{AChE}$ activity was significantly correlated with MOA activity $(\mathrm{r}=0.62 ; \mathrm{p}<0.001)$.

Oxidative stress and metallothionein (MT) levels were also determined in brain tissue (Figures 2A and 2B). FHM were exposed to the $\mathrm{ME}$, nano- $\mathrm{ZnO}$ and $\mathrm{ZnCl}_{2}$ alone and in combination. The levels of brain MT (A) and LPO levels (B) were reported. The letter $a$ indicates significant from controls and $b$ significant between malesfemales at a given treatment condition. Factorial analysis of variance revealed that MT levels in brain were significantly affected by $\operatorname{sex}^{\star}$ effluent/Zn treatments interaction. The levels of MT were higher in females compared to males in unexposed fish. This trend was lost upon exposure to combination of municipal and either forms of dietary Zn. Brain MT levels were increased by exposure to the municipal effluent at the lowest concentration (5\%). Neither dietary nano- $\mathrm{ZnO}$ nor $\mathrm{ZnCl}_{2}$ significantly induced MT in male and female fish. Exposure to the combination of municipal and either forms of dietary Zn significantly decreased brain MT levels. Correlation analysis revealed that brain 


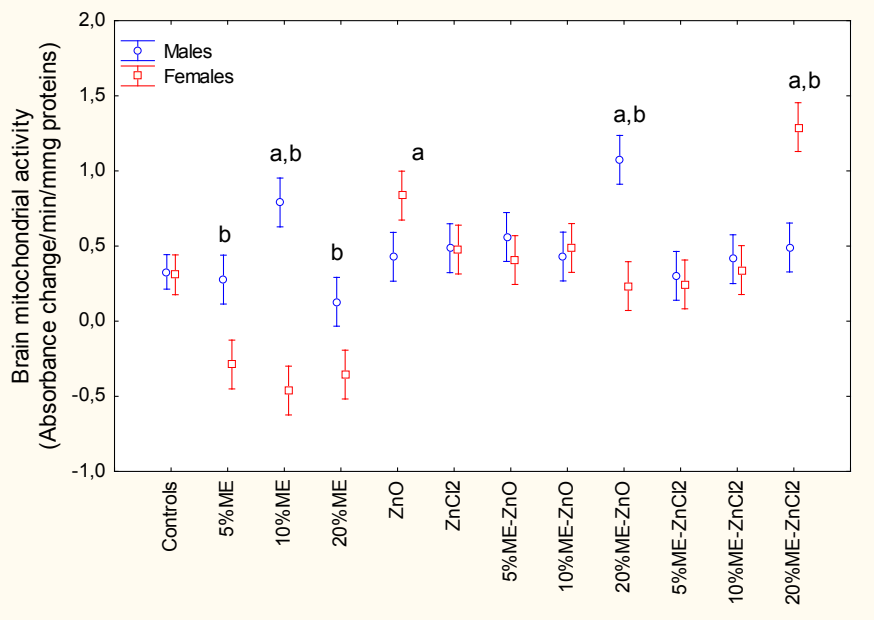

Exposure conditions

Figure 2. Oxidative stress and metallothioneins in fish brain.

FHM were exposed to the $\mathrm{ME}$, nano- $\mathrm{ZnO}$ and $\mathrm{ZnCl} 2$ alone and in combination. The levels of brain MT (A) and LPO levels (B) were reported. The letter a indicates significant from controls and $\mathrm{b}$ significant between males-females at a given treatment condition.

MT levels were significantly correlated with condition factor $(\mathrm{r}=-0.33$; $\mathrm{p}=0.001)$, BSI $(\mathrm{r}=-0.36 ; \mathrm{p}<0.001), \mathrm{AChE}(\mathrm{r}=0.2 ; \mathrm{p}<0.05), \mathrm{MAO}(\mathrm{r}=0.29$; $\mathrm{p}<0.01), \mathrm{Fe}(\mathrm{r}=-0.28 ; \mathrm{p}<0.01)$, Co $(\mathrm{r}=-0.27 ; \mathrm{p}<0.01), \mathrm{Cu}(\mathrm{r}=-0.21$; $\mathrm{p}<0.05), \mathrm{Ag}(\mathrm{r}=-0.25 ; \mathrm{p}<0.05)$ and $\mathrm{Cd}(\mathrm{r}=-0.21 ; \mathrm{p}<0.05)$. Lipid-mediated oxidative damage was also determined using the thiobarbituric acid reactants assay (Figure $2 \mathrm{~B}$ ). Factorial analysis of variance revealed a significant effluent/Zn treatments ${ }^{*}$ sex interaction. Brain LPO levels were significantly higher in males compared to females in control fish. Male LPO levels were increased at $10 \%$ municipal effluent alone, 10 and $20 \% \mathrm{ME}$ with dietary nano- $\mathrm{ZnO}$ treatment groups. There were no difference in LPO levels between males and females in control fish but levels in males were significantly lower at $20 \%$ municipal effluent and $10,20 \%$ municipal effluent in fish fed a nano- $\mathrm{ZnO}$-supplemented feed. Correlation analysis revealed that brain LPO levels were significantly correlated with condition factor $(\mathrm{r}=0.29 ; \mathrm{p}<0.01)$, BSI $(\mathrm{r}=0.55$; $\mathrm{p}<0.001)$, MT $(\mathrm{r}=-0.30 ; \mathrm{p}<0.01), \operatorname{AChE}(\mathrm{r}=0.37 ; \mathrm{p}<0.001)$, MAO $(\mathrm{r}=0.36 ; \mathrm{p}<0.001), \mathrm{Ni}(\mathrm{r}=-0.31 ; \mathrm{p}<0.01), \mathrm{Cu}(\mathrm{r}=-0.35 ; \mathrm{p}<0.001), \mathrm{Zn}(\mathrm{r}=-$ $0.44 ; \mathrm{p}<0.001)$ and $\mathrm{Cd}(\mathrm{r}=0.25 ; \mathrm{p}<0.05)$.

Cellular metabolic energy expenditure was determined by measuring brain mitochondrial electron transport (MET) activity (Figure 3). FHM were exposed to municipal effluents, nano- $\mathrm{ZnO}$ and $\mathrm{ZnCl}_{2}$ alone and in combination, and cellular energy expenditure was determined following MET activity as described in Methods. The letter a indicates significant from controls and b significant between malesfemales at a given treatment condition. Factorial analysis of variance indicated that all factors were significant with effluent/Zn treatments ${ }^{*}$ sex interaction. MET activity was similar in females and males. It was decreased in females exposed to municipal effluent alone and increased in males exposed to $10 \%$ municipal effluent. Dietary nano-ZnO slightly increased MET activity in females. The combination of $20 \%$ municipal effluent with fish fed nano-ZnO-spiked feed increased MET activity in males while the combination of the $20 \%$ municipal effluent with fish fed a $\mathrm{ZnCl}_{2}$-spiked feed increased MET activity in females. Correlation analysis revealed that MET activity was significantly correlated with brain LPO $(r=0.33 ; \mathrm{p}<0.001)$, MT $(\mathrm{r}=-0.31 ; \mathrm{p}<0.01)$, DOP $(\mathrm{r}=-0.46 ; \mathrm{p}<0.01)$, and $\mathrm{Cu}(\mathrm{r}=-021 ; \mathrm{p}<0.05)$. The extent of DNA damage was determined using the alkaline DNA precipitation assay (Figure 4). FHM were exposed to the $\mathrm{ME}$, nano- $\mathrm{ZnO}$ and $\mathrm{ZnCl}_{2}$ alone
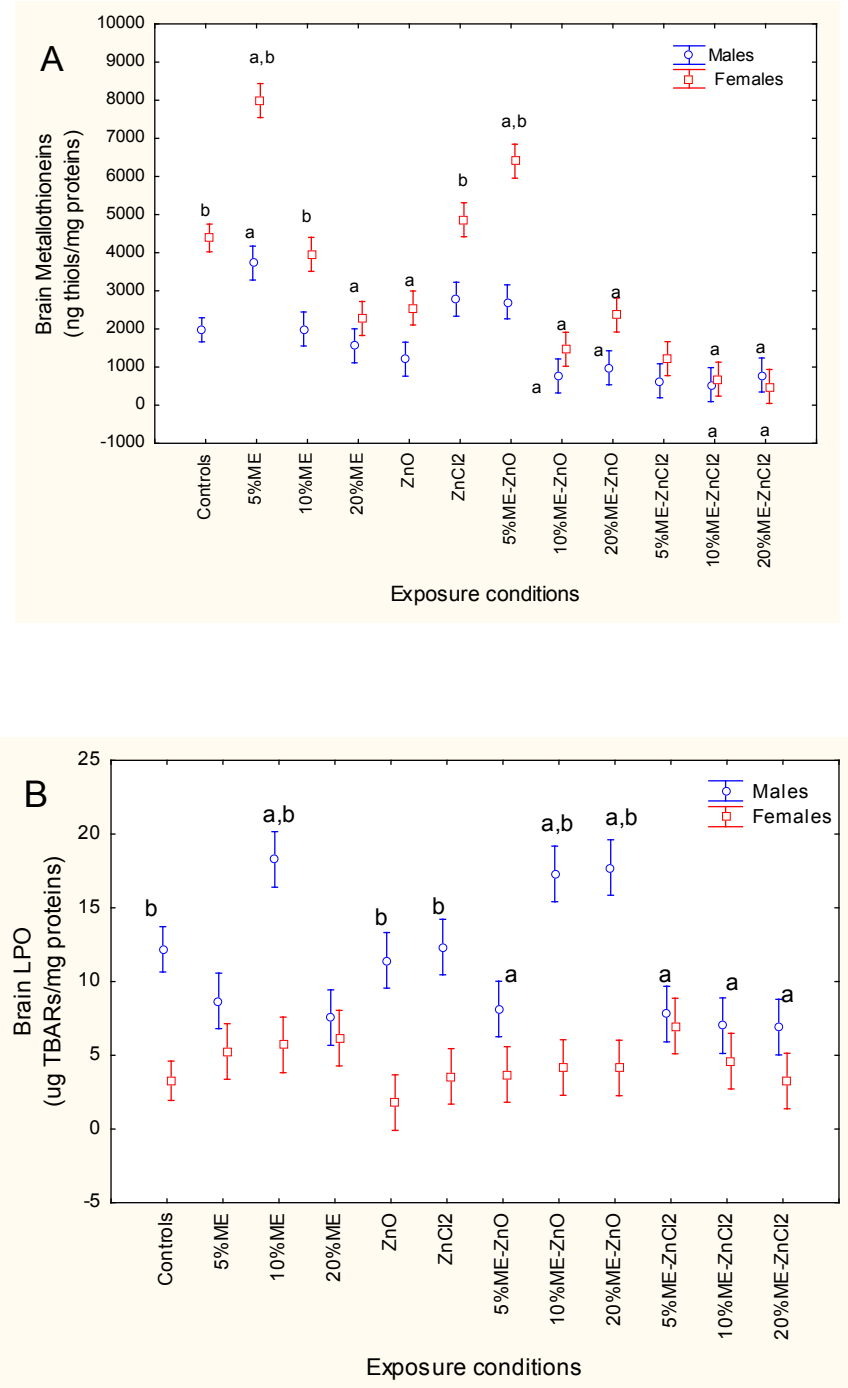

Figure 3. Brain energy expenditure in fish exposed to $\mathrm{ME}$, nano- $\mathrm{ZnO}$ and $\mathrm{ZnCl}_{2}$.

FHM were exposed to municipal effluents, nano- $\mathrm{ZnO}$ and $\mathrm{ZnCl}_{2}$ alone and in combination, and cellular energy expenditure was determined following MET activity as described in Methods. The letter a indicates significant from controls and $\mathrm{b}$ significant between malesfemales at a given treatment condition.

and in combination and DAN strand breaks in brain homogenates were determined as described in Methods. The letter a indicates significant from controls and b significant between males-females at a given treatment condition. Factorial analysis of variance revealed a significant effluent/Zn treatments ${ }^{*}$ sex interaction. Baseline DNA strand breaks were similar between males and females and DNA strand breaks decreased at high municipal effluent concentration. Neither the dietary forms of $\mathrm{Zn}$ produced changes in DNA strand breaks levels. The combination of municipal effluent and nano- $\mathrm{ZnO}$ or $\mathrm{ZnCl}_{2}$ produced no significant changes. Correlation analysis revealed that brain DNA strand breaks were significantly correlated with LPO $(r=0.4 ; p<0.001)$, $\operatorname{AChE}(r=0.25 ; p=0.01), \operatorname{MET}(r=0.36 ; p<0.001), \mathrm{Cu}(\mathrm{r}=-0.30 ; \mathrm{p}<0.01)$ and $\mathrm{Zn}(\mathrm{r}=-0.29 ; \mathrm{p}<0.01)$.

In the attempt to obtain a global view on the impacts of municipal effluent, nano- $\mathrm{ZnO}$ or $\mathrm{ZnCl}_{2}$ supplemented feed alone and in combination, a discriminant function and factorial analysis was performed (Figure 5). The data were analyzed to seek out general 


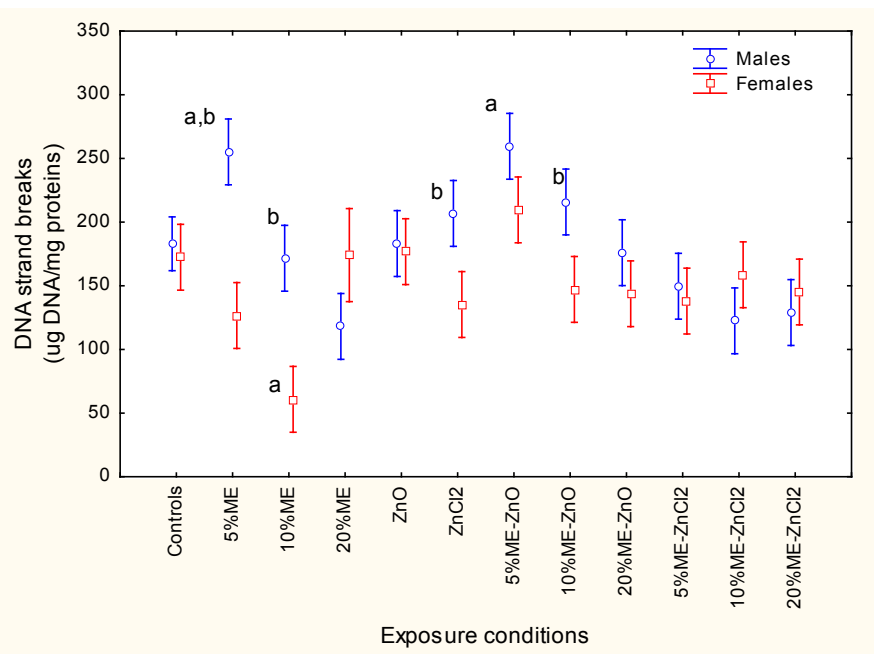

Figure 4. Brain DNA damage in fish exposed to $\mathrm{ME}$, nano- $\mathrm{ZnO}$ and $\mathrm{ZnCl}_{2}$.

FHM were exposed to the ME, nano- $\mathrm{ZnO}$ and $\mathrm{ZnCl}_{2}$ alone and in combination and DAN strand breaks in brain homogenates were determined as described in Methods. The letter a indicates significant from controls and $\mathrm{b}$ significant between males-females at a given treatment condition.

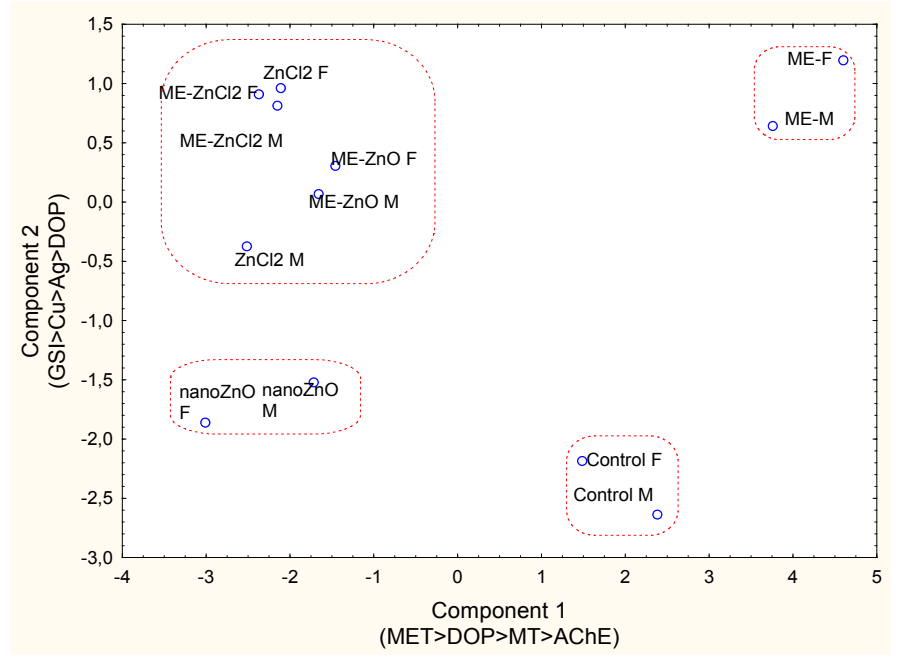

Figure 5. Discriminate function and factorial analyses of biomarker data.

The data were analyzed to seek out general classes of responses and identified the most important biomarkers. Mean site classification was $70 \%$ and 4 clusters were identified. The biomarkers identified at the $\mathrm{x}$ and $\mathrm{y}$ axes are those with the high factorial weights. The effects of dietary nano- $\mathrm{ZnO}$ and the municipal effluent could be identified by the biomarkers on the $\mathrm{x}$ axis.

classes of responses and identified the most important biomarkers. Mean site classification was $70 \%$ and 4 clusters were identified. The biomarkers identified at the $\mathrm{x}$ and $\mathrm{y}$ axes are those with the high factorial weights. The effects of dietary nano- $\mathrm{ZnO}$ and the municipal effluent could be identified by the biomarkers on the $\mathrm{x}$ axis. Because gender was often a significant factor in the observed responses, it was included in the analysis as well. The mean classification efficiency was $70 \%$ which suggests good separation of the various treatments between the municipal effluent, nano- $\mathrm{ZnO}$ and $\mathrm{ZnCl}_{2}$ alone and in combination. The analysis generated four distinct clusters of treatments. The first cluster consisted of control males and females. The second cluster consisted of male and female fish exposed to the municipal effluent. The third cluster consisted of male and female fish fed a nano- $\mathrm{ZnO}$ diet.
The 4th cluster consisted of male and female fish fed a $\mathrm{ZnCl}_{2}$ diet alone and municipal effluent exposed fish fed either a nano- $\mathrm{ZnO}$ or $\mathrm{ZnCl}_{2}$ diet. This cluster suggests that when municipal effluent is present, the effects on fish fed a nano-ZnO-supplemented feed were similar to those in fish fed a $\mathrm{ZnCl}_{2}$ diet.

\section{Discussion}

In this study, fathead minnows were fed a daily intake of $3 \mu \mathrm{g}$ dietary $\mathrm{Zn}$ and continuously exposed to increasing concentrations of municipal effluent for 21 days. Total $\mathrm{Zn}$ levels in fish carcasses did not significantly change across all exposure treatments. This suggests that $\mathrm{Zn}$ was not accumulated in fish tissue with the feeding regime used after 21 days at $25^{\circ} \mathrm{C}$, under which the fish were exposed to two modes of entry: dietary uptake and waterborne exposure. This is consistent with a recent study that found that dietary $\mathrm{Cu}$ and $\mathrm{Zn}$ did not accumulate in the heart, brain or muscle tissue of goldfish [31]. Dietary nano-ZnO were shown to accumulate primarily in the intestines, followed by the gills and liver. Neither brain MT nor LPO levels were significantly affected, which suggests that neither form of dietary $\mathrm{Zn}$ overwhelmed normal $\mathrm{Zn}$ homeostasis. However, dietary nano- $\mathrm{ZnO}$ and $\mathrm{ZnCl}_{2}$ significantly decreased brain MAO activity, which suggests decreased turnover of monoamines in the central nervous system. There is little in the scientific literature on the neurotoxicological effects of $\mathrm{Zn}$ nanoparticles in fish. In another study, waterborne exposure of goldfish to suspensions of nano- $\mathrm{ZnO}$ resulted in accumulation in the gills and gut, with only a small fraction reaching more internal tissues, such as the liver and muscle tissue [17]. Moreover, nano- $\mathrm{ZnO}$ partitioned in metal-rich granules in the liver with no significant mobilization of $\mathrm{Zn}$ to the MT compartment (cytosol), suggesting that nano- $\mathrm{ZnO}$ could be found in cells and partition in insoluble granules. The lack of induction of MT suggests that nano- $\mathrm{ZnO}$ is rather stable in the liver and does not liberate $\mathrm{Zn}^{2+}$. The lack of MT response and superoxide dismutase activity was also found in medaka fish exposed to nano- $\mathrm{ZnO}$, but heat shock protein $70 \mathrm{mRNA}$ levels were up-regulated, indicating a different mode of action for nano- $\mathrm{ZnO}$ effects independent of $\mathrm{Zn}$ cation release [32]. However, nano- $\mathrm{ZnO}$ seemed to increase oxidative stress in the carp Cyprinus carpio in the brain, liver and gills, but at much higher concentrations than that used in the present study (500 to 50000 $\mu \mathrm{g} / \mathrm{L}$ ) [33]. Waterborne exposure to nano- $\mathrm{ZnO}$ at these concentrations decreased thiol levels and increased LPO in the organs described above. Exposure to the effluent increased brain dopamine levels, MAO activity and AChE activity, whereas dietary uptake of nano- $\mathrm{ZnO}$ and $\mathrm{ZnCl}_{2}$ eliminated these effects. MAO activity was readily decreased by either form of $\mathrm{Zn}$ compared to the controls, whereas the presence of municipal effluent had no effect. Brain AChE and liver superoxide dismutase were significantly decreased by nano- $\mathrm{ZnO}$ in the goldfish Carassius auratus [34]. These effects suggests that lower brain and liver metabolic activity occurred, but only at high concentrations of nano$\mathrm{ZnO}(160$ and $320 \mathrm{mg} / \mathrm{L})$ after 4 days.

Exposure to municipal effluents had more significant effects in fish than the relatively low concentrations of dietary nano- $\mathrm{ZnO}$ or $\mathrm{ZnCl}_{2}$. Indeed, exposure to 12 municipal effluents led to increased expression of MT and oxidative stress markers at the mRNA levels [35]. Increased MT levels were reported in brown trout exposed to sewage treatment plant effluent, suggesting the presence of labile divalent metals [36]. Municipal effluent on its own, produced significant changes in tissue $\mathrm{Fe}, \mathrm{Co}, \mathrm{Cu}, \mathrm{Ag}$ and $\mathrm{Cd}$ levels and had no bearing effect on $\mathrm{Zn}$ tissue levels. Caged fish place downstream of a municipal effluent outfall led to increased oxidative stress, such as LPO and protein carbonyls [37]. Brain LPO levels were elevated in males exposed to municipal effluent 
in the presence of dietary nano- $\mathrm{ZnO}$, but not in the presence of $\mathrm{ZnCl}_{2}$. The latter form of $\mathrm{Zn}$ is considered an antioxidant essential metal [38] while nano- $\mathrm{ZnO}$ can lead to oxidative stress in fish brain [33]. Increased oxidative stress was correlated with DNA strand breaks, suggesting that oxidative damage contributed to DNA strand breaks. Indeed, an analysis of covariance of brain DNA strand breaks with brain LPO as the covariate revealed that LPO contributed the most to DNA strand breaks and was no longer induced in fish exposed to both the municipal effluent and nano- $\mathrm{ZnO}$. This supports the hypothesis that nano- $\mathrm{ZnO}$ induced genotoxicity in fish exposed to municipal effluents is mediated by oxidative stress. The genotoxicity of municipal effluents could also result from altered DNA metabolism and stability [8]. Decreased DNA strand breaks were related to inhibition of purine synthesis by inhibition of dehydrofolate reductase activity, a key enzyme in purine synthesis in mussels exposed to the same type of municipal effluent [13]. Exposure to municipal effluent could also lead to more severe cytogenetic damage such as in micronuclei, as shown in RTG-2 cells exposed to such effluents [39]. The etiology of micronuclei formation is difficult to determine with complex mixtures, but the reported levels of bisphenol A, octylphenol and phthalates alone were not able to explain the observed genotoxicity of the effluents. The same was observed in rainbow trout exposed to a municipal effluent whose levels in oil products $\left(\mathrm{C}_{14-28}\right)$, benzo(a)pyrene, heavy metals and suspended solids did not exceed the maximal permissible concentration for wastewaters, with the fish showing elevated incidence of micronuclei in blood and decreased macrophage counts [40]. Pharmaceutical products also contribute to the genotoxicity of the effluents [41]. Exposure of Danio rerio to realistic concentrations (i.e. those found in Italian municipal effluents) of sildenafil, atorvastatin and gemfibrozil for one week led to DNA damage. Genotoxicity was determined by the Comet assay and showed loss of DNA integrity in fish exposed to the above pharmaceuticals.

The increased levels of dopamine and AChE activity in fish exposed to municipal effluent is consistent with catecholamineinduced neuroactivity, associated with conditioned place preference behaviour [22]. The addition of nano- $\mathrm{ZnO}$ to the diet decreased AChE in females exposed to the municipal, suggesting decreased condition place preference behaviour. It would have been of interest to monitor this behaviour in females exposed to 10 and $20 \%$ of municipal effluent and dietary nano- $\mathrm{ZnO}$ in relation to the time spent near the spawning tiles. The estrogenic effects of municipal effluents could also have significant effects on fish brain and behaviour. Indeed, the brain is also a source of estradiol, with the young brain exhibiting increased E2 synthesis, cell proliferation and neurogenesis [42]. Exposure of fish to E2 increased monoamine oxidase activity and decreased serotonin levels in developing tilapia brains, which suggests a negative interaction between E2 and serotonin levels [43]. Exposure of E2 and phytoestrogens at environmentally realistic concentrations increased brain dopamine levels in Betta splendens [44]. The increase in brain dopamine was located at the forebrain, but the application of therapeutic doses of phytoestrogens decreased dopamine levels. Exposure to E2 resulted in an increase in dopamine D2 receptor mRNA, which is involved in dopamine-induced inactivation of nerve cells and could represent a mechanism that protects dopaminergic neurons from toxic injury [45]. Increased dopaminergic activity was related to oocyte recruitment, which is negatively regulated by the expression of D2 dopamine receptors. The increase in dopamine D2 receptors was followed by decreased follicle-stimulating hormone (growth of ovarian follicles) and luteinizing hormone (involved in steroidogenesis) for steroidogenesis and oocyte growth. The increase in D2 dopamine receptors is consistent with decreased neuron energy expenditure (MET) in females, and the reported decreased egg production by municipal effluents reported elsewhere [46]. Interestingly, the inclusion of nano- $\mathrm{ZnO}$ and $\mathrm{ZnCl}_{2}$ in the diet eliminated the decrease in MET activity in fish exposed to municipal effluent. In conclusion, exposure of adult fathead minnow to a physicochemically treated municipal leads to changes in brain dopamine levels and MAO, MET and AChE activity [47]. The addition of nano- $\mathrm{ZnO}$ and $\mathrm{ZnCl}_{2}$ reversed the fish response to municipal effluents for dopamine, MAO and AchE in the brain, indicating a negative interaction between exposure to municipal effluent and dietary nano- $\mathrm{ZnO}$ and $\mathrm{ZnCl}_{2}$. On the whole, the fish responses to combined exposure of municipal effluent and either forms of $\mathrm{Zn}$ were more closely correlated with dietary $\mathrm{ZnCl}_{2}$-induced effects, suggesting that dietary nano- $\mathrm{ZnO}$ effects are primarily explained by $\mathrm{ZnCl}_{2}$ in either the absence or presence of municipal effluents.

\section{Acknowledgements}

The authors thank Sophie Trépanier for running the fathead minnow exposure experiments. The work was funded by the Chemical Management Plan and the St. Lawrence Action Plan of Environment Canada.

\section{References}

1. Hadioui M, Leclerc S, Wilkinson KJ (2013) Multimethod quantification of Ag+ release from nanosilver. Talanta 105: 15-19. [Crossref]

2. Kundu P, Anumol EA, Ravishankar N (2013) Pristine nanomaterials: synthesis, stability and applications. Nanoscale 5: 5215-5224. [Crossref]

3. Shi LE, Li ZH, Zheng W, Zhao YF, Jin YF, et al. (2014) Synthesis, antibacterial activity, antibacterial mechanism and food applications of $\mathrm{ZnO}$ nanoparticles: a review. Food Addit Contam Part A Chem Anal Control Expo Risk Assess 31: 173-186. [Crossref]

4. Li M, Pokhrel S, Jin X, Mädler L, Damoiseaux R, et al. (2011) Stability, bioavailability, and bacterial toxicity of $\mathrm{ZnO}$ and iron-doped $\mathrm{ZnO}$ nanoparticles in aquatic media. Environ Sci Technol 45: 755-761. [Crossref]

5. Gagné F, Auclair J, Peyrot C, Wilkinson KJ (2015) The influence of zinc chloride and zinc oxide nanoparticles on air-time survival in freshwater mussels. Comp Biochem Physiol C Toxicol Pharmacol 172-173: 36-44. [Crossref]

6. Keller AA, Wang H, Zhou D, Lenihan HS, Cherr G, et al. (2010) Stability and aggregation of metal oxide nanoparticles in natural aqueous matrices. Environ Sci Technol 44: 1962-1967. [Crossref]

7. Holeton C, Chambers P A, Grace L. 2011. Wastewater release and its impacts on Canadian waters. Can.J.Fish.Aquat.Sci. 68, 1836-1859.

8. Gagné F, André C, Cejka P, Hausler R, Fournier M (2011) Alterations in DNA metabolism in Elliptio complanata mussels after exposure to municipal effluents. Comp Biochem Physiol C Toxicol Pharmacol 154: 100-107. [Crossref]

9. Arstikaitis J, Gagné F, Cyr DG (2014) Exposure of fathead minnows to municipal wastewater effluent affects intracellular signaling pathways in the liver. Comp Biochem Physiol C Toxicol Pharmacol 164: 1-10. [Crossref]

10. Leet JK, Sassman S, Amberg JJ, Olmstead AW, Lee LS, et al. (2015) Environmental hormones and their impacts on sex differentiation in fathead minnows. Aquat Toxicol 158: 98-107. [Crossref]

11. Gagné F, Blaise C, Hellou J (2004) Endocrine disruption and health effects of caged mussels, Elliptio complanata, placed downstream from a primary-treated municipal effluent plume for one year. Comp Biochem Physiol 138C: 33-44.

12. Aravindaksham J, Paquet V, Gregory M, Dufresne, J, Fournier, et al. (2004) Consequences of Xenoestrogen Exposure on Male Reproductive Function in Spottail Shiners (Notropis hudsonius). Toxicological Sciences 78: 156-165.

13. Lajeunesse A, Gagnon C, Gagné F, Louis S, Cejka P, et al. (2011) Distribution of antidepressants and their metabolites in brook trout exposed to municipal wastewaters before and after ozone treatment--evidence of biological effects. Chemosphere 83: 564571. [Crossref]

14. Tetreault GR, Bennett J, Cheng C, Servos, MR, McMaster ME (2012) Reproductive 
and histopathological effects in wild fish inhabiting an effluent-dominated stream, Wascana Creek, SK, Canada. Aquatic Toxicology 110-111: 149-161.

15. Lacaze E, Devaux A, Bony S, Bruneau A, André C, et al. (2013) Genotoxic impact of a municipal effluent dispersion plume in the freshwater mussel Elliptio complanata: an in situ study. J Xenobiotics 3: 14-16.

16. Dubey A, Goswami M, Yadav K, Chaudhary D (2015) Oxidative Stress and NanoToxicity Induced by $\mathrm{TiO} 2$ and $\mathrm{ZnO}$ on WAG Cell Line. PLoS One 10: e0127493. [Crossref]

17. Fan W, Li Q, Yang X, Zhang L (2013) Zn subcellular distribution in liver of goldfish (carassius auratus) with exposure to zinc oxide nanoparticles and mechanism of hepatic detoxification. PLoS One 8: e78123. [Crossref]

18. Shariati F, Shariati S (2011) Review on methods for determination of metallothioneins in aquatic organisms. Biol Trace Elem Res 141: 340-366. [Crossref]

19. Menelaou E, Udvadia AJ, Tanguay RL, Svoboda KR (2014) Activation of $\hat{I} \pm 2 A$ containing nicotinic acetylcholine receptors mediates nicotine-induced motor output in embryonic zebrafish. Eur J Neurosci 40: 2225-2240. [Crossref]

20. Kim YH, Lee Y, Kim D, Jung MW, Lee CJ (2010) Scopolamine-induced learning impairment reversed by physostigmine in zebrafish. Neurosci Res 67: 156-161. [Crossref]

21. Leal E, Fernández-Durán B, Agulleiro MJ, Conde-Siera M, Míguez JM, et al. (2013) Effects of dopaminergic system activation on feeding behavior and growth performance of the sea bass (Dicentrarchus labrax): a self-feeding approach. Horm Behav 64: 113121. [Crossref]

22. Ninkovic J, Folchert A, Makhankov YV, Neuhauss SC, Sillaber I, et al. (2006) Genetic identification of $\mathrm{AChE}$ as a positive modulator of addiction to the psychostimulant D-amphetamine in zebrafish. $J$ Neurobiol 66: 463-475. [Crossref]

23. Bonacci S, Browne MA, Dissanayake A, Hagger JA, Corsi I, et al. (2004) Esterase activities in the bivalve mollusc Adamussium colbecki as a biomarker for pollution monitoring in the Antarctic marine environment. Mar Pollut Bull 49: 445-455.

24. Gagné F (2014) Neuroendocrine disruption. In: Biochemical Ecotoxicology-Principles and Methods. First Edition, Chapter 9, Elsevier Inc USA: 145-170.

25. Wills ED (1987) Evaluation of lipid peroxidation in lipids and biological membranes In: Snell K, Mullock B. (Eds.), Biochemical Toxicology: A Practical Approach. IRL Press, Washington, USA:127.

26. Olive PL (1988) DNA precipitation assay: a rapid and simple method for detecting DNA damage in mammalian cells. Environ Mol Mutagen 11: 487-495. [Crossref]

27. Viarengo A, Ponzanon E, Dondero F, Fabbri R (1997) A simple spectrophotometric method for metallothionein evaluation in marine organisms: an application to Mediterranean and Antarctic molluscs. Mar Environ Res 44: 69-84.

28. Haase H, Maret W (2004) A differential assay for the reduced and oxidized states of metallothionein and thionein. Anal Biochem 333: 19-26. [Crossref]

29. Smolders R, Bervoets L, De Coen W, Blust R (2004) Cellular energy allocation in zebra mussels exposed along a pollution gradient: linking cellular effects to higher levels of biological organization. Environ Poll 129: 99-112.

30. King F, Packard TT (1975) Respiration and the activity of the respiratory electron transport system in marine zooplankton. Limnol. Oceanog. 20: 849-854.

31. Ates M, Arslan Z, Demir V, Daniels J, Farah IO (2015) Accumulation and toxicity of $\mathrm{CuO}$ and $\mathrm{ZnO}$ nanoparticles through waterborne and dietary exposure of goldfish (Carassius auratus). Environ Toxicol 30: 119-128. [Crossref]
32. Wong SW, Leung PT, DjurisiÄł AB, Leung KM (2010) Toxicities of nano zinc oxide to five marine organisms: influences of aggregate size and ion solubility. Anal Bioanal Chem 396: 609-618. [Crossref]

33. Hao L, Chen L (2012) Oxidative stress responses in different organs of carp (Cyprinus carpio) with exposure to $\mathrm{ZnO}$ nanoparticles. Ecotoxicol Environ Saf 80: 103-110. [Crossref]

34. Xia J, Zhao HZ, Lu GH (2013) Effects of selected metal oxide nanoparticles on multiple biomarkers in Carassius auratus. Biomed Environ Sci 26: 742-749

35. Gagné F, Smyth SA, André C, Douville M, Gélinas M, et al. (2013) Stress-related gene expression changes in rainbow trout hepatocytes exposed to various municipal wastewater treatment influents and effluents. Environ Sci Pollut Res Int 20: 1706-1718. [Crossref]

36. Burkhardt-Holm P, Bernet D, Hogstrand C (1999) Increase of metallothioneinimmunopositive chloride cells in the gills of brown trout and rainbow trout after exposure to sewage treatment plant effluents. Histochem J 31: 339-346. [Crossref]

37. Carney Almroth B, Albertsson E, Sturve J, Förlin L (2008) Oxidative stress, eviden in antioxidant defences and damage products, in rainbow trout caged outside a sewage treatment plant. Ecotoxicol Environ Saf 70: 370-378. [Crossref]

38. Formigari A, Irato P, Santon A (2007) Zinc, antioxidant systems and metallothionein in metal mediated-apoptosis: biochemical and cytochemical aspects. Comp Biochem Physiol C Toxicol Pharmacol 146: 443-459. [Crossref]

39. Llorente MT, Parra JM, Sánchez-Fortún S, Castaño A (2012) Cytotoxicity and genotoxicity of sewage treatment plant effluents in rainbow trout cells (RTG-2). Water Res 46: 6351-6358. [Crossref]

40. VosylienÄ MZ, KazlauskienÄ N, BarÅ $i$ ienÄ J, AndreikÄ nait Ä L, MilukaitÄ A, et al (2010) Ecotoxicity and genotoxicity relating to fish in wastewaters discharged from the Vilnius treatment plant. Water Sci Technol 62: 859-865. [Crossref]

41. Rocco L, Frenzilli G, Zito G, Archimandritis A, Peluso C, et al. (2012) Genotoxic effects in fish induced by pharmacological agents present in the sewage of some Italian water-treatment plants. Environ Toxicol 27: 18-25. [Crossref]

42. Lin CJ, Fan-Chiang YC, Dufour S, Chang CF.Activation of brain steroidogenesis and neurogenesis during the gonadal differentiation in protandrous black porgy, Acanthopagrus schlegelii. Dev Neurobiol. In press.

43. Tsai CL, Wang LH (1999) Effects of gonadal steroids on the serotonin synthesis and metabolism in the early developing tilapia brain. Neurosci Lett 264: 45-48. [Crossref]

44. Clotfelter ED, McNitt MM, Carpenter RE, Summers CH (2010) Modulation of monoamine neurotransmitters in fighting fish Betta splendens exposed to waterborne phytoestrogens. Fish Physiol Biochem 36: 933-943. [Crossref]

45. Levavi-Sivan B, Biran J, Fireman E (2006) Sex steroids are involved in the regulation of gonadotropin-releasing hormone and dopamine D2 receptors in female tilapia pituitary. Biol Reprod 75: 642-650. [Crossref]

46. Lister A, Regan C, Van Zwol J, Van Der Kraak G (2009) Inhibition of egg production in zebrafish by fluoxetine and municipal effluents: a mechanistic evaluation. Aqua Toxicol 95: 320-329. [Crossref]

47. Bradford MM (1976) A rapid and sensitive method for the quantitation of microgram quantities of protein utilizing the principle of protein-dye binding. Anal Biochem 72 248-254. [Crossref]

Copyright: (C2016 Gagné F. This is an open-access article distributed under the terms of the Creative Commons Attribution License, which permits unrestricted use, distribution, and reproduction in any medium, provided the original author and source are credited. 